\title{
Monte-Carlo fluid approaches to detached plasmas in non-axisymmetric divertor configurations
}

\author{
Y. Feng ${ }^{1)}$, H. Frerichs ${ }^{2)}$, M. Kobayashi ${ }^{3)}$, D. Reiter ${ }^{4)}$ \\ ${ }^{1)}$ Max-Planck-Institut für Plasma Physik, 17491 Greifswald, Germany \\ ${ }^{2)}$ Department of Engineering Physics, University of Wisconsin - Madison, Madison, WI 53706, USA \\ ${ }^{3)}$ National Institute for Fusion Science, 322-6 Oroshi-cho, Toki 509-5292, Japan \\ ${ }^{4)}$ Forschungszentrum Jülich GmbH, Institut für Energie- und Klimaforschung - Plasmaphysik, 52425 Jülich, Germany
}

\begin{abstract}
Fluid transport modeling in three-dimensional (3D) boundaries of toroidal confinement devices is reviewed with the emphasis on a Monte-Carlo approach to simulate detached plasmas. The loss of axisymmetry in such configurations presents a major challenge for numerical implementation of the standard fluid model widely applied to fusion experimental devices. A large-scale effort has been made to address this problem under complementary aspects including different magnetic topologies and numerical techniques. In this paper, we give a brief review of the different strategies pioneered and the challenges involved. A more detailed description is provided for the Monte-Carlo code EMC3-Eirene, where the physics model and the basic idea behind the applied Monte-Carlo method are presented. The focus is put on its applications to detachment studies for stellarators and tokamaks. Here, major achievements and difficulties encountered are described. Model limitations and further development plans are discussed.
\end{abstract}

\section{Introduction}

Developing a 3D fluid model for understanding the edge plasma transport in helical devices was initially triggered by the first island divertor experiments at W7-AS [1] and has been further promoted by the divertor activities on LHD [2,3] and the island divertor program planned for W7-X [4]. The ELM-control potential of resonant magnetic perturbations (RMP) demonstrated at DIII-D [5] and other existing tokamaks $[6,7]$ has attracted strong interest from ITER [8]. The RMP-fields break the axisymmetry of the poloidal divertor, therefore motivating the need of 3D numerical tools.

The fluid model widely used for understanding edge plasma transport consists of a set of Braginskii's equations [9]. Mathematically, they fall in the category of second-order partial differential equations of parabolic type. Numerical solutions of these equations in 3D magnetic fields first encounter the difficulty of establishing an appropriate coordinate system to allow for a clean separation of the highly-anisotropic transport, which usually needs to be field-aligned. In axisymmetric configurations smooth flux surfaces exist and provide a natural coordinate for the finite-difference/volume-based 2D models developed for tokamaks [10-12]. In general, magnetic field lines in a non-axisymmetric configuration do not form flux surfaces everywhere, but usually exhibit a certain degree of stochastic behavior. For most of the practical 3D cases, it is thus difficult to find a rule to order the stochastic field lines into a mesh acceptable for the conventional methods. Despite these difficulties, great effort has been made to address the 3D transport problems by utilizing the well-developed finite-volume/difference methods. Typical examples are the BoRiS [13] and FINDIF [14] codes. The former is based on a finite-volume method in generalized magnetic 
coordinates, while the latter is a finite-difference code formulated in local magnetic coordinates associated with a quite complicated grid generation process.

Monte-Carlo methods, by contrast, provide a completely different approach to this problem. The basic idea behind this approach arose from the fact that the fluid equations can be generalized into a Fokker-Planck form which is equivalent to the Langevin equation originating from describing Brownian motion, also known as stochastic equation in the literature. The stochastic equation can be integrated locally without the need of a continuum of the computational grid, which is the fundamental idea behind the E3D [15] and EMC3 [16] codes.

All edge plasma codes require some form of neutral gas model for a self-consistent treatment of plasma-neutral and plasma-wall interaction processes, and one such model is provided by the Eirene code [17]. This paper focuses on the EMC3-Eirene code, in particular on its applications to detachment studies for 3D divertors. After a brief introduction to the code in the next section, we present its applications to detachment studies for tokamaks and stellarators in section 3. Model limitations, technical challenges and further development plans are discussed in section 4. A short summary is given in section 5 .

\section{The EMC3-Eirene code}

The development of the Edge Monte-Carlo 3D EMC3 code begun with a rather simple fluid model including only the heat conduction process for electrons, taking into account, from the very beginning, the fully 3D edge structure of magnetic configurations and divertor installations, however. Thus, code applications to specific devices were possible and were done during the whole code development process. The physics model in the code was improved gradually by separating the energy transport between ions and electrons, by implementing the momentum balance and continuity equations, by coupling the Eirene code for neutral transport and by including a trace-impurity fluid model. Both EMC3 and Eirene are fully parallelized.

Numerically, the EMC3 code employs a new advanced Monte Carlo technique for the treatment of highly anisotropic 3D fluid transport processes which are aligned to local field lines of finite lengths. The Monte-Carlo method does not need a continuum of the computational grid, but a continuum of field-lines to prevent the fast parallel transport from entering the perpendicular transport channels (the so-called numerical diffusion effect). This is guaranteed by developing a new reversible field line mapping technique [18].

The present EMC3 code version [19] solves a set of time-independent fluid equations for the mass, momentum and energy for electrons and ions (hydrogen isotopes) given as

$$
\begin{aligned}
& \vec{\nabla} \cdot\left(n_{i} V_{i l l} \overrightarrow{\boldsymbol{b}}-D \overrightarrow{\boldsymbol{b}_{\perp}} \overrightarrow{\boldsymbol{b}}_{\perp} \cdot \vec{\nabla} n_{i}\right)=S_{p} \\
& \vec{\nabla} \cdot\left(m_{i} n_{i} V_{i l l} V_{i l l} \overrightarrow{\boldsymbol{b}}-\eta_{l l} \overrightarrow{\boldsymbol{b}} \overrightarrow{\boldsymbol{b}} \cdot \vec{\nabla} V_{i l l}-D \overrightarrow{\boldsymbol{b}_{\perp}} \overrightarrow{\boldsymbol{b}}_{\perp} \cdot \vec{\nabla} m_{i} n_{i} V_{i l l}\right)=-\overrightarrow{\boldsymbol{b}} \cdot \vec{\nabla} p+S_{m} \\
& \vec{\nabla} \cdot\left(\frac{5}{2} n_{e} T_{e} V_{i l l} \overrightarrow{\boldsymbol{b}}-\kappa_{e} \overrightarrow{\boldsymbol{b}} \overrightarrow{\boldsymbol{b}} \cdot \vec{\nabla} T_{e}-\frac{5}{2} T_{e} D \overrightarrow{\boldsymbol{b}}_{\perp} \overrightarrow{\boldsymbol{b}_{\perp}} \cdot \vec{\nabla} n_{e}-\chi_{e} n_{e} \overrightarrow{\boldsymbol{b}_{\perp}} \overrightarrow{\boldsymbol{b}_{\perp}} \cdot \vec{\nabla} T_{e}\right)=-k\left(T_{e}-T_{i}\right)+S_{e e} \\
& \vec{\nabla} \cdot\left(\frac{5}{2} n_{i} T_{i} V_{i l l} \overrightarrow{\boldsymbol{b}}-\kappa_{i} \overrightarrow{\boldsymbol{b}} \overrightarrow{\boldsymbol{b}} \cdot \vec{\nabla} T_{i}-\frac{5}{2} T_{i} D \overrightarrow{\boldsymbol{b}}_{\perp} \overrightarrow{\boldsymbol{b}}_{\perp} \cdot \vec{\nabla} n_{i}-\chi_{i} n_{i} \overrightarrow{\boldsymbol{b}}_{\perp} \overrightarrow{\boldsymbol{b}}_{\perp} \cdot \vec{\nabla} T_{i}\right)=+k\left(T_{e}-T_{i}\right)+S_{e i}
\end{aligned}
$$

where $\boldsymbol{b}$ is the unit vector of the B-field line and $\boldsymbol{b}_{\perp} \boldsymbol{b}_{\perp}=\boldsymbol{I}-\boldsymbol{b} \boldsymbol{b}$, with $\mathbf{I}$ being the unit tensor. $\boldsymbol{\kappa}_{e}$ and $\boldsymbol{\kappa}_{i}$ are the Spitzer heat conductivity for electrons and ions. $S_{p}, S_{m}, S_{e e}$ and $S_{e i}$ are the particle, momentum 
and energy sources associated with neutrals. In addition, $S_{e e}$ includes also the energy loss from impurity radiation. The other terms and symbols have their usual meanings.

In addition the EMC3 code includes a trace impurity transport model consisting of continuity

$$
\vec{\nabla} \cdot\left(n_{I}^{z} V_{I l l}^{z} \overrightarrow{\boldsymbol{b}}-D_{I}^{z} \overrightarrow{\boldsymbol{b}}_{\perp} \overrightarrow{\boldsymbol{b}}_{\perp} \cdot \vec{\nabla} n_{I}^{z}\right)=S_{z-1 \rightarrow z}-S_{z \rightarrow z+1}+R_{z+1 \rightarrow z}-R_{z \rightarrow z-1}
$$

and of a simplified momentum balance

$$
\begin{aligned}
& \frac{m_{I} m_{i}\left(V_{I I I}^{z}-V_{i l l}\right)}{\tau_{s}}=-\overrightarrow{\boldsymbol{b}} \cdot \vec{\nabla} n_{I}^{z} T_{i}+n_{I}^{z} Z e E_{l l}+0.71 n_{I}^{z} Z^{2} \overrightarrow{\boldsymbol{b}} \cdot \vec{\nabla} T_{e}+n_{I}^{z} \beta \overrightarrow{\boldsymbol{b}} \cdot \vec{\nabla} T_{i} \\
& \text { with } \beta_{i}=3 \frac{\mu+5 \sqrt{2} Z^{2}\left(1.1 \mu^{5 / 2}-0.35 \mu^{3 / 2}\right)-1}{2.6-2 \mu+5.4 \mu^{2}} \text { where } \mu=\frac{m_{I}}{m_{I}+m_{i}}
\end{aligned}
$$

for each ionization stage $Z . m_{I}$ and $m_{i}$ are the respective impurity and bulk ion mass. The four terms on the right side of equation (5) represent the ionization and recombination processes. The term on the left side of equation (6) is the friction with plasma ions and the last two terms on the right side are the thermal forces. The parallel E-field $E_{/ /}$is determined by the parallel momentum equation for electrons:

$$
\overrightarrow{\boldsymbol{b}} \cdot \vec{\nabla} n_{e} T_{e}+n_{e} e E_{l \mid}+0.71 n_{e} \overrightarrow{\boldsymbol{b}} \cdot \vec{\nabla} T_{e}=0
$$

In addition, $T_{Z}=T_{i}$ for all $Z$ has been assumed. Within the trace impurity transport model, there is no limitation on the number of impurity species, but a strict restriction on the impurity concentration. Impurities influence the background plasma via radiation, only. Charge contribution and the counter forces exerted on the background plasma as well as the interaction among the different ionization stages are completely ignored.

The fluid equations are generalized into a common Fokker-Planck form [16, 19], which is solved by applying a Monte-Carlo method in flux-tube coordinates in combination with the reversible fieldline mapping technique [18]. Each flux tube is formed by four field lines and requires convex crosssections everywhere along the field lines. This is only possible for sufficiently short field lines because of finite magnetic shear effects. In most of the practical cases, the flux tubes satisfying the convex condition are usually shorter than required by the configuration periodicity of a device. Thus, the entire computational domain has to be divided into several toroidal segments (sub-domains), with each having its own toroidal span angle $\Delta \phi$ and sharing a common $\phi-$ plane with its neighbor. Each sub-domain is filled with a large number of finite flux tubes without gaps and overlaps in real space. Depending on the actual configuration, the flux tubes in each toroidal sub-domain can be grouped and ordered into a set of standard blocks $[20,21]$. A standard block means that the field-lines (flux tubes) there can be labeled by two independent indices and the adjacent field lines in the labeling index are also neighbors in real space. Neighboring blocks in real space must share common field lines on the interface. The continuum of field lines is then guaranteed by the reversible field line mapping technique. This strategy of geometry and transport treatment makes the fluid modeling feasible for most of the edge magnetic configurations explored in fusion devices. 
The EMC3-Eirene code is in continuous development. In recent years the code has been improved in many aspects, including 1) the implementation of a noise-free particle-splitting technique to improve the Monte Carlo statistic in low-temperature ranges of most interest [22], 2) implicit coupling to a 1D core impurity transport model to remove the ad hoc boundary conditions for intrinsic impurities at the SOL-core interface [23], 3) mesh structure optimization for complex divertor configurations [20], 4) introduction of non-uniform cross-field field transport coefficients $[22], 5)$ separation between axisymmetric and non-axisymmetric plasma-facing components to accelerate the neutral transport simulation in tokamaks [22] and 6) a model extension to helium plasma [24].

Thanks to its high geometric flexibility as well as its 3D capability, the EMC3-Eirene has been applied to a wide variety of magnetic confinement fusion devices including stellarators [25-30], tokamaks [31-47] and linear devices [48, 49], bridging different divertor concepts.

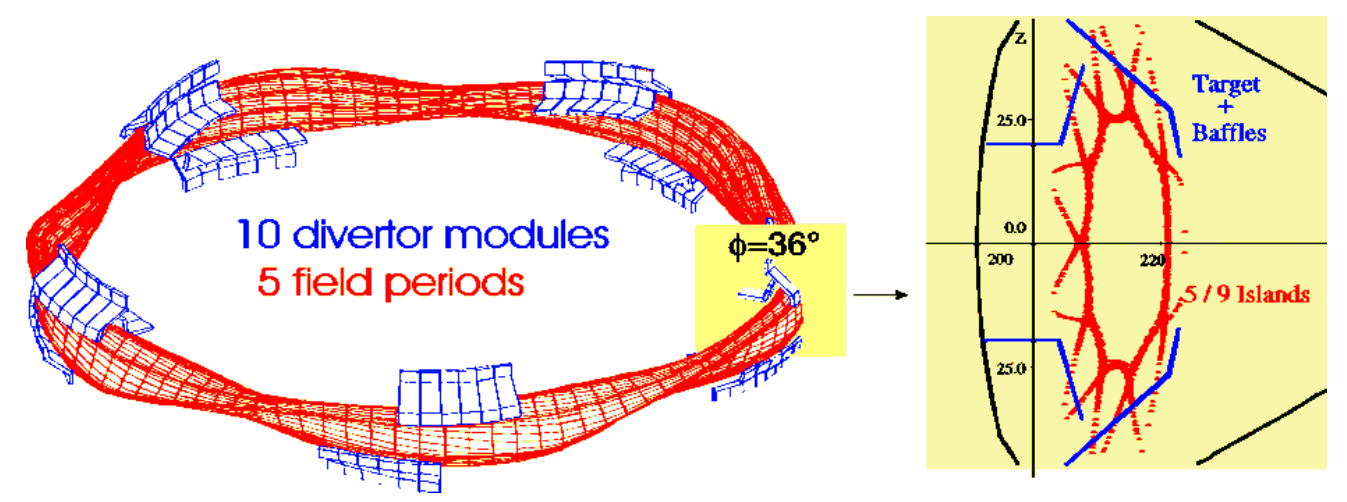

Figure 1. Island divertor of W7-AS. Ten up/down symmetric divertor modules are installed around the elliptical planes. The so-called standard divertor configuration is based on the $t=5 / 9$ island chain.

\section{Applications to detached plasmas}

\section{1 stellarators}

3.1.1 W7-AS. W7-AS was a low-shear stellarator having a major radius of $2 \mathrm{~m}$ and a minor radius of $0.14-0.20 \mathrm{~m}$ depending on the position of plasma-limiting components installed. It was operated with limiters until 2000. In the last eighteen months before its shutdown in 2002, a dedicated island divertor program [1] was conducted to explore the divertor potential of low-order magnetic islands for controlling plasma exhaust in low-shear stellarators - the so-called island divertor. The island divertor operation significantly improved the recycling conditions in W7-AS, making the plasma density easily controllable even in the presence of a strong NBI-source. Divertor operation was accompanied by the discovery of a new confinement regime, the HDH (High-Density-H) -mode [50], which is characterized by high density with good energy and low impurity confinement. Stationary partial detachment was achieved with intense impurity radiation localized at the plasma edge. The major scientific achievements at W7-AS have been summarized in [51, 52].

Basically, the island divertor follows the same principle as the tokamak poloidal-field divertor [53]. The magnetic islands either have a naturally open diverting structure or are opened by intersecting 
target plates. The plasma, which enters the islands across the inner separatrix, is guided by the open field lines inside the islands towards the targets positioned at the outer separatrix (Figure 1). The islands form an intermediate scrape-off layer (SOL) between the confinement core and the plasmasurface interaction region, preventing the core plasma from a direct exposure to the recycling neutrals and the sputtered impurities. Large differences between tokamaks and W7-AS exist, however, in the respective magnetic and divertor geometries. While the conventional poloidal divertor in tokamaks relies on a poloidal field of roughly 10 percent of the total field, the island divertor in W7-AS (in W7-X as well) is based on a resonant radial field component of the order of $10^{-3}$. The former can be operated with single- or double-null, whereas the latter has a multi-null geometry (nine X-points in W7-AS). Limited by the island size, the target plates in W7-AS are much

EMC3-EIRENE simulations
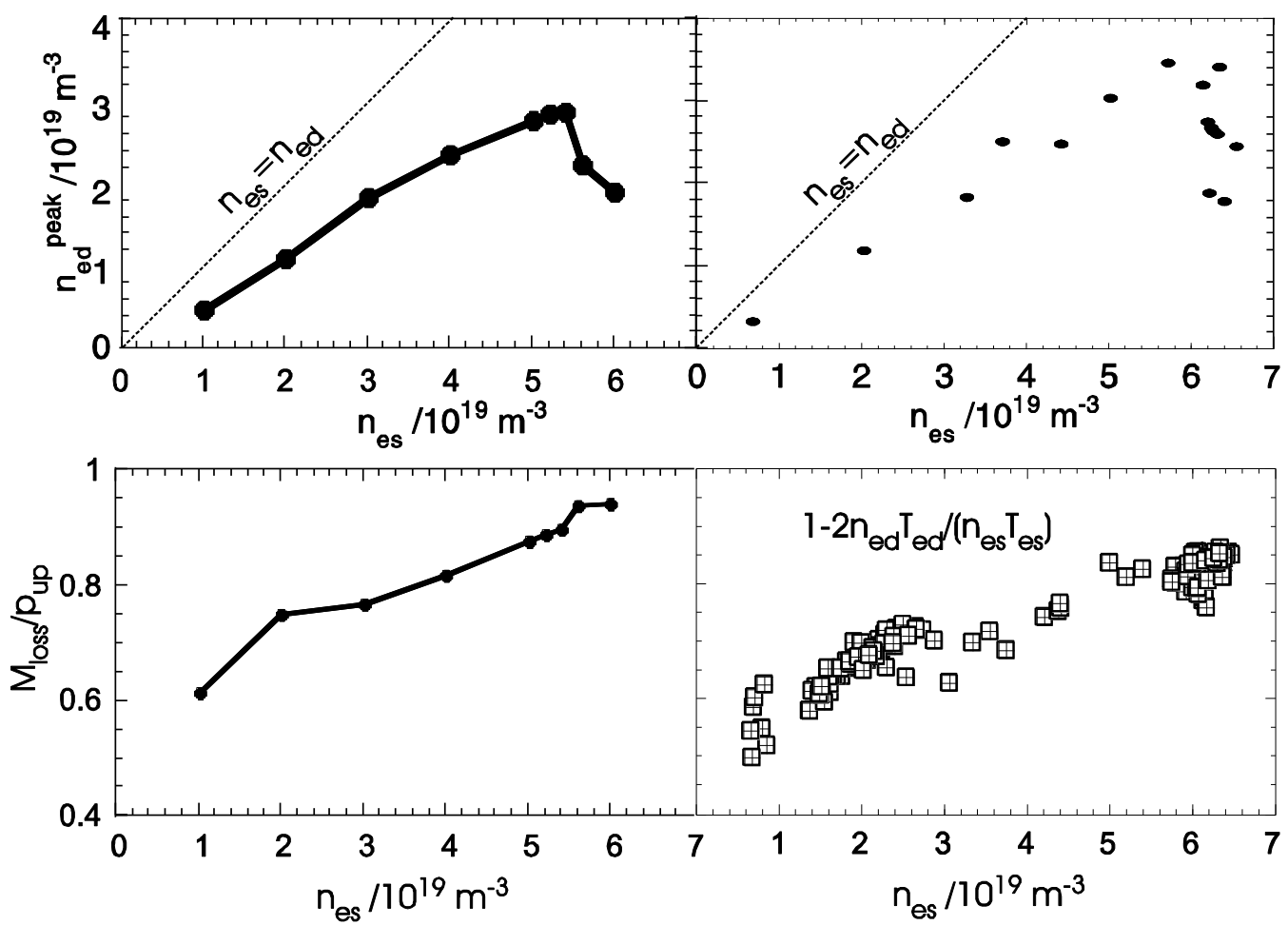

Figure 2. A density-scan up to detachment. Top: Correlations between target density $n_{\mathrm{ed}}$ and separatrix density $\mathrm{n}_{\mathrm{es}}$ calculated by EMC3-Eirene (left) and measured by target Langmuir probes (right) for hydrogen plasmas. Bottom: momentum loss as a function of $n_{\text {es }}$ from simulations and experiments. In the experiments, a pressure balance analysis is only possible for electrons.

closer to the confinement region than those in tokamaks. All these differences make the behavior of the detached plasmas in the island divertor deviate from that realized in tokamaks. Detachment simulations made for W7-AS mainly focused on understanding the basic physics/transport processes in detachment associated with the island divertor geometry, rather than attempting to quantitatively reproduce any local measurement because of the absence of drifts in the EMC3-Eirene code and the use of vacuum fields.

Route to detachment. First detachment simulations predicted that the W7-AS divertor plasma goes into detachment without a prior high-recycling regime [54], which has indeed been confirmed experimentally. Figure 2 shows the EMC3-Eirene simulation results [55] for a density scan up to 
detachment, compared with Langmuir probe measurements. The power into the SOL, $\mathrm{P}_{\mathrm{SOL}}$, is estimated to be $1 \mathrm{MW}$ at low densities and gradually dropped to $0.85 \mathrm{MW}$ at detachment due to increased core radiation at high densities. $\mathrm{P}_{\mathrm{SOL}}$ is equally allocated to electrons and ions. An anomalous particle diffusivity of $D=0.5 \mathrm{~m}^{2} / \mathrm{s}$ is assumed, and the electron and ion heat conductivity is three times $D$. Both transport coefficients are spatially constant.

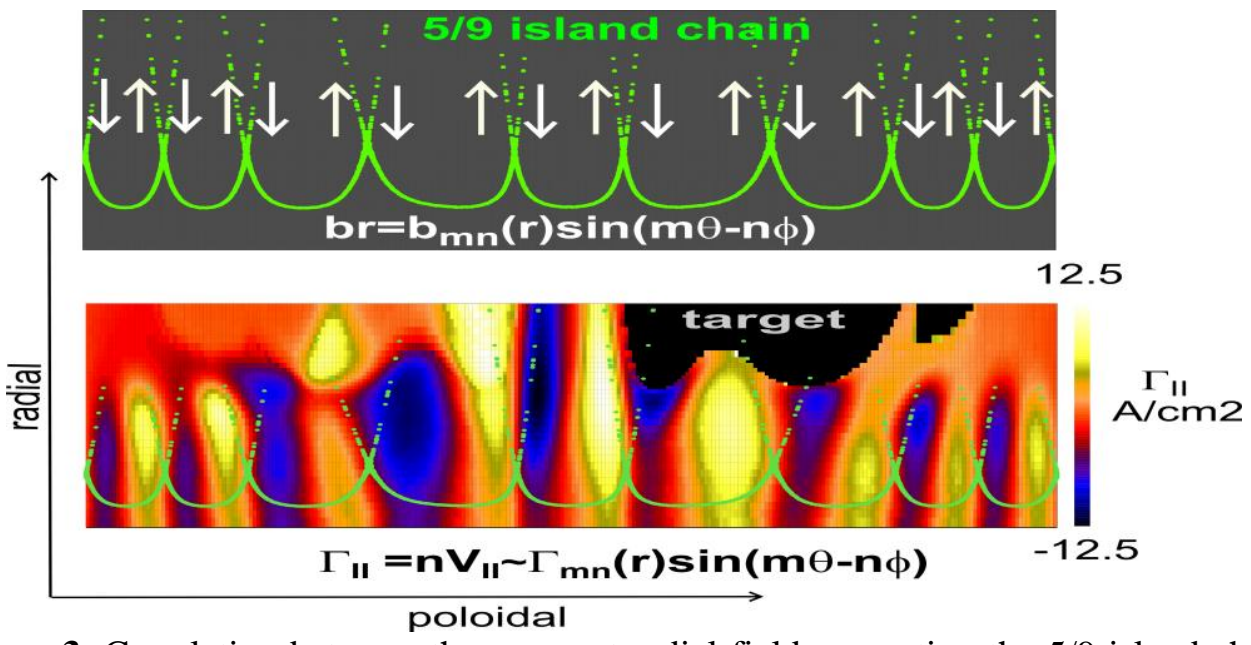

Figure 3. Correlation between the resonant radial field generating the $5 / 9$ island chain (top) and the parallel particle flux density calculated by EMC3-Eirene (bottom). The plots are made in mesh coordinates based on nested, smooth, closed surfaces.

The measured electron density $n_{e d}$ and temperature $T_{e d}$ are the peak values from the Langmuir probes mounted on the target segments where the plasma first detaches with increasing density [1]. In the simulations, they are the averaged values within the strike zones weighted by the plasma thermal pressure at the targets for statistical reasons associated with the Monte-Carlo scheme adopted. The momentum loss is defined by $M_{\text {loss }}=P_{u p}-2 P_{d}$, where $P_{u p}$ is the total ion and electron thermal pressure at the separatrix (LCFS), while $P_{d}$ is the same at the targets. In the code results, $P_{u p}$ is averaged on the LCFS due to the complex structure of the 3D plasma flow patterns. This average is reasonable as the Mach numbers there are usually small. In experiments, a pressure balance is established based on local electron density and temperature measurements.

The target density $n_{e d}$ increases almost linearly with rising $n_{e s}$ up to a rollover point and then drops once the plasma goes into detachment. A coincident drop was also observed for the recycling flux/ion saturation current in both simulation and experiment. Note that $n_{e d}$ remains everywhere smaller than $n_{e s}$. The rollover of the target density and recycling flux, as well as the absence of a prior high-recycling regime, are attributed to a cross-field viscous momentum transport associated with the island geometry. This process causes significant momentum losses already at low densities without intensive plasma-neutral interaction (the lower picture in figure 2). The neutral contribution has been estimated by switching off the corresponding terms in the 3D code and turned out to play a negligible role. After transition to detachment, the ionization front separates from the target, causing a radial inward extension of the parallel flows, thereby enhancing the viscous momentum loss [55].

Viscous momentum loss. The $t=5 / 9$ island chain in W7-AS is generated by a resonant radial field $b_{r}=b_{m n} \sin (m \theta-n \phi)$ with $m=9$ and $n=5$ being the respective poloidal and toroidal mode numbers, which is schematically sketched in the top picture in figure 3. Parallel transport across the islands toward the targets is guided by $b_{r}$, resulting in a parallel particle flux distribution which is closely 
correlated with the radial field structure (figure 3). The parallel particle flux $\Gamma_{\| l}=n V_{\|}\left(\mathrm{n}=\mathrm{n}_{\mathrm{e}}=\mathrm{n}_{\mathrm{i}}\right)$ has roughly the same phase distribution as $b_{r}$. The periodic change in the sign of $b_{r}$ induces counterflows in such a way as to result always in a positive radial particle flux, i.e. $\Gamma_{r}=b_{r} \Gamma_{l /} \sim \sin ^{2}(m \theta-n \phi)>$ 0 , driven by the radial plasma pressure drop. Associated with small island size (radial $\times$ poloidal $\sim 3-4$ $\mathrm{cm} \times 9 \mathrm{~cm})$ and the long connection length $(\sim 100 \mathrm{~m}$ from target to target) in W7-AS, the parallel flow channels are poloidally broad enough to have contact with each other. Radially, they can even propagate across the LCFS into the confinement region (figure 3). The friction between the counterflows and with the core-plasma is considered to be the main momentum loss mechanism. In addition, the momentum transported into the shadowed regions of the discontinuously placed target plates by means of diffusion makes an additional contribution.

Detachment stability. W7-AS divertor experiments have shown that the stability of a detached plasma depends on the island size $(\Delta x)$ and the connection length $\left(\mathrm{L}_{\mathrm{c}}\right)$ [56], which can be externally controlled by the island control coils. The island control coils provide an additional resonant perturbation field to enlarge the islands and to shorten the $\mathrm{L}_{\mathrm{c}}$ therein. Figure 4 shows the locations of stable detachment in a $\Delta \mathrm{x}-\mathrm{L}_{\mathrm{c}}$ diagram. Stable detached plasmas were achieved with sufficientlylarge island control coil currents $\mathrm{I}_{\mathrm{cc}}$ (maximal $3.5 \mathrm{kA}$ ). Without the control coils, establishing a stable detachment in the

island divertor would be impossible. These experimental findings motivated a detailed numerical analysis to understand the physics behind the geometry-related detachment stability [57], for which four configurations/discharges were selected (figure 4). In the computations, $\mathrm{P}_{\mathrm{SOL}}$ was varied in the range from 0.8 to $1.2 \mathrm{MW}$ and $\mathrm{D}$ from 0.5 to $1 \mathrm{~m}^{2} / \mathrm{s}$ to ensure that the major results are not affected by the uncertainties in these input parameters. The intrinsic carbon was assumed as the only impurity species.

Detachment simulations are performed by raising the separatrix (upstream) density $\mathrm{n}_{\mathrm{es}}$, similarly to the

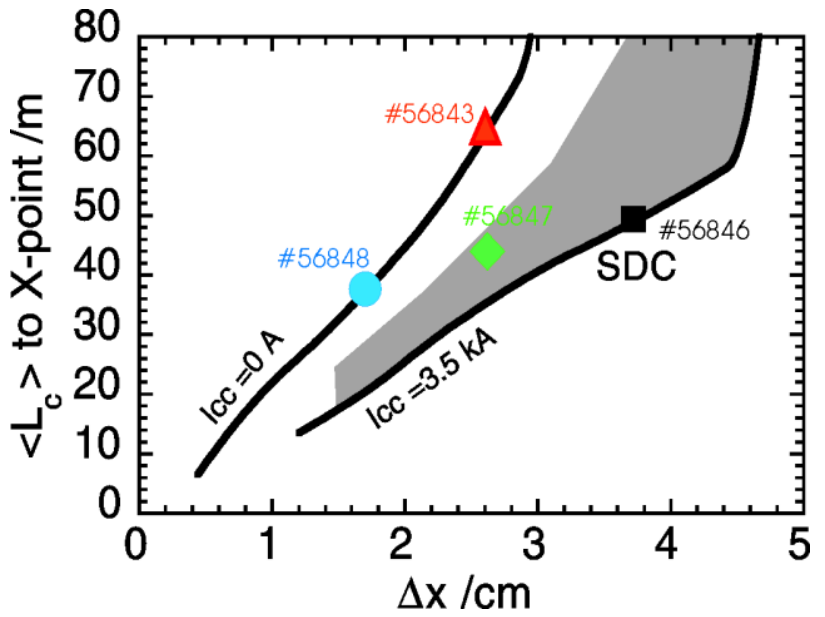

Figure 4. Stable partial detachment is restricted to large $\Delta \mathrm{x}$ (island size measured by the target-to-X-point distance at $\phi=27^{\circ}$ ) and small $\mathrm{L}_{\mathrm{c}}$ ranges (shadowed region). The four symbols indicate four discharges selected for numerical studies. SDC: standard divertor configuration.

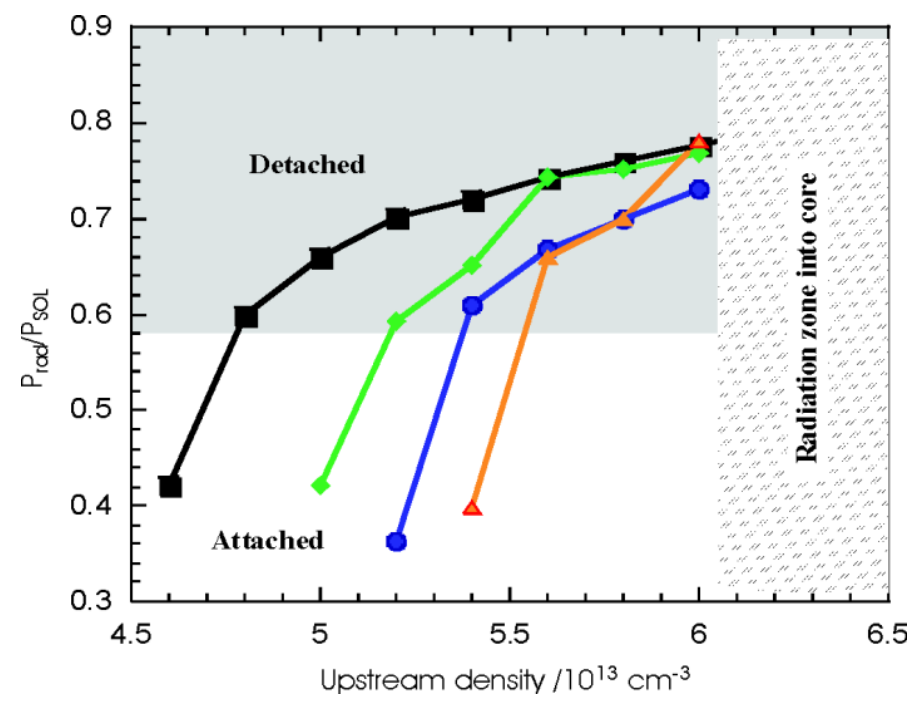

Figure 5. Carbon radiation normalized to $\mathrm{P}_{\text {sol }}$ as a function of $\mathrm{n}_{\mathrm{es}}$ and configuration. The meaning of the different symbols can be found in figure 4 . 


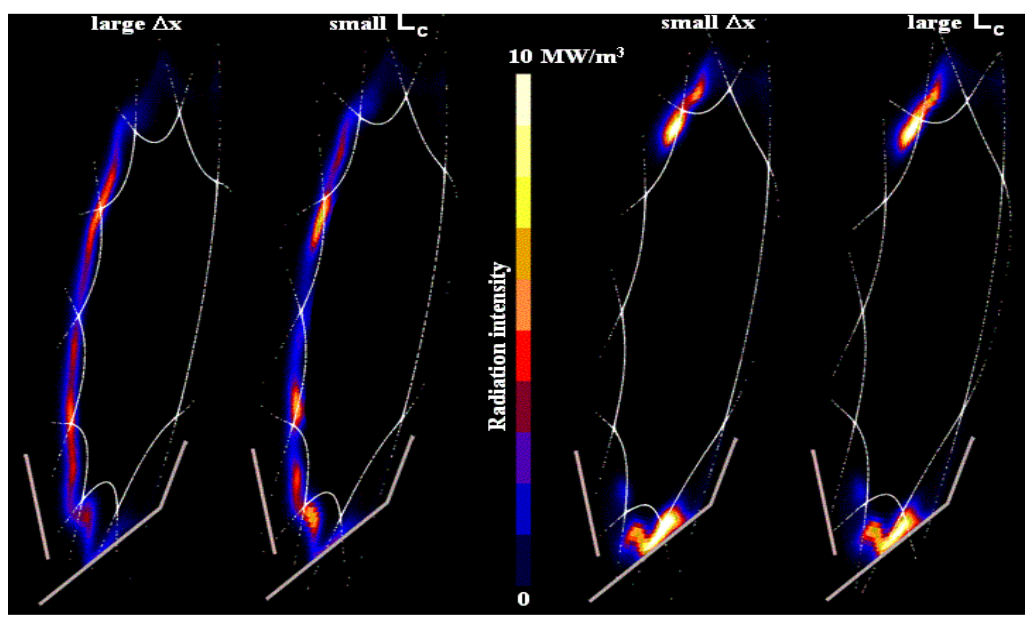

Figure 6. Two typical radiation patterns are identified by the code: Left: Inboard side radiation for large $\Delta \mathrm{x}(\# 56846)$ and small $\mathrm{L}_{\mathrm{c}}(\# 56847)$ Right: Divertor radiation for small $\Delta \mathrm{x}(\# 56848)$ or large $\mathrm{L}_{\mathrm{c}}(\# 56843)$

density ramp in the divertor experiments. Then, $\mathrm{n}_{\mathrm{es}}$ is further increased in small steps until the radiation zone shifts into the core. Figure 5 shows the dependence of carbon radiation on $\mathrm{n}_{\mathrm{es}}$ for the four selected configurations. By decreasing $\Delta \mathrm{x}$ or enlarging $\mathrm{L}_{\mathrm{c}}$, the detachment transition shifts to higher densities, which agrees well with the general experimental tendency [57]. In addition, figures 4 and 5 show also that by decreasing $\Delta x$ or enlarging $L_{c}$ the $n_{e s}$-window of detachment becomes narrower.

The simulations for the four configurations produced two different radiation patterns, namely inboard-side radiation and divertor radiation (figure 6). For the two stable configurations with finite $\mathrm{I}_{\mathrm{cc}}$ the radiation is concentrated around inner X-points, while an intense radiation zone is formed in the divertor region for the other two unstable configurations with $\mathrm{I}_{\mathrm{cc}}=0$. The formation of the two different radiation patterns has not yet been fully understood. In what follows we give an intuitive explanation based on the EMC3-Eirene model. The flux surfaces of W7-AS are compressed on the outboard side and expanded on the inboard side. This explains why most of the W7-AS islands reside on the inboard side. As a consequence, more heat sources enter the SOL on the outboard side. Thus, in general, impurity radiation prefers the inboard side. In the two $\mathrm{I}_{\mathrm{cc}}=0$ cases, either a small $\Delta \mathrm{x}(\# 56848)$ or a large $\mathrm{L}_{\mathrm{c}}$

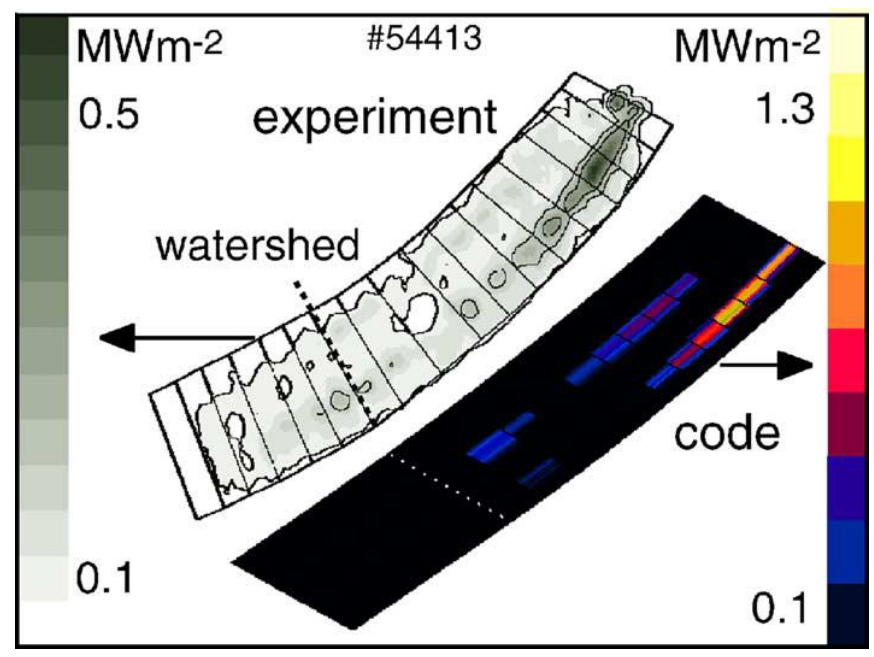

Figure 7. Power deposition patterns from thermography for a partially detached discharge and simulation by the EMC3EIRENE code. 
(\#56843) would increase the weight of cross-field transport and thereby the relative thickness of the radiation layer to the island size. If the former would become comparable to or exceed the latter, the heat transport from the outboard side to the inboard side for sustaining inboard-side radiation would be hindered by the intercepting targets within the power channel. This hypothesis is supported by the observation that the two different radiation pictures converge into one radiation pattern, i.e. an intensive radiation zone appearing on the inboard mid-plane once they enter the confinement region by further increasing $\mathrm{n}_{\mathrm{es}}$ in the simulations. In this case, the plasma becomes unstable experimentally and a MARFE-like radiation originating from that region was indeed observed by CCD cameras [58, 59].

The inboard-side radiation picture predicted for the stable discharges is supported by target thermography measurements which detected a hotspot on the target location magnetically closer to the outboard side during a stable detachment phase [60] (figure 7). This is the reason why the stable detachment achieved on W7-AS is termed "partial detachment". A similar hotspot was also produced by the EMC3-Eirene code (figure 7).

The radiation locations have a direct consequence on the island screening efficiency of the recycling neutrals. The divertor radiation location for the two $\mathrm{I}_{\mathrm{cc}}=0$ configurations cools the recycling zone, making the islands transparent for the recycling neutrals. The Eirene code shows that, in this case, the neutral penetration flux into the core is much more sensitive to the SOL power and the separatrix density than in the inboard-side radiation case. A linear stability analysis shows that the penetrating neutrals can drive a thermal instability [57]. The loss of the island neutral screening is considered to be the reason for the detachment instability observed in experiments with insufficient island control coil currents.

Open issues. In spite of the similar tendencies in the leading-order effects shown by experiment and modeling on W7-AS, there still remain numerous experimental details which numerically are not recovered. For example, asymmetries in divertor plasmas between the top and bottom divertor modules, and among the strike zones as well, were usually observed in detached plasmas $[60,61]$. The asymmetries correlate with the B-field direction and thus are considered to be driven by classical drifts. Associated with the up/down asymmetry, spectrometers have measured a high ratio of $\mathrm{H}_{\gamma}$ and $\mathrm{H}_{\alpha}$ emission lines originating from the divertor module with a higher neutral pressure, indicating the existence of a localized recombination zone [62, 63]. A self-consistent treatment of the drifts is beyond the present code capability. All detachment simulations made for W7-AS were made without volume recombination processes. An estimate by post-processing the 3D simulation results for W7-AS shows a negligible role of volume recombination in comparison with surface recombination processes, however.

3.1.2 LHD. The LHD is a heliotron-type device. The divertor plates and the first wall are made of graphite and stainless steel, respectively. The magnetic field is produced by superconducting coils with a poloidal winding number of $l=2$ and toroidal field periods of $n=10$ [3]. The major radius and the averaged minor radius of the plasma (including the stochastic layers outside the last closed flux surface (LCFS)) are $3.9 \mathrm{~m}$ and $0.7 \mathrm{~m}$, respectively. Because of the mode spectrum of the magnetic field produced by the helical coils, magnetic islands of different mode numbers are created, and they overlap each other. There exists, therefore, intrinsically a stochastic field line structure in the edge region. The simulation of detachment in LHD has been often performed for discharges with 
RMP (resonant magnetic perturbation) application, where the detachment phase becomes rather stable and can easily be sustained for the duration of NBI heating (typically several seconds) [64]. The RMP has mode numbers $m / n=1 / 1$ and creates a magnetic island in the stochastic layer. In this case, the computational domain covers one half of the torus, and the other half of the torus is reproduced by using the stellarator symmetry. In this way, the $n=1$ mode structure is reproduced in the computation. In the radial direction, the computational domain starts a few millimeters inside the LCFS and covers the entire stochastic layer. The grid resolution in the radial direction, $\Delta r$, is typically a few millimeters. In the toroidal direction, $\phi, \Delta \phi=1$ degree [65]. A finer resolution with $\Delta \phi=0.25$ degrees is used when the divertor legs are fully resolved [21]. This finer resolution is necessary to avoid destruction of the cell volume of the field aligned grid caused by the strong magnetic shear in the edge region in LHD. The poloidal direction is usually divided into 600 cells. In this section, the results of the simulations and the comparison with the experiments are presented.

Figure 8 (a) shows the divertor particle flux (total recycling flux in the whole computation domain) as a function of plasma density at the LCFS, $\mathrm{n}_{\mathrm{LCFS}}$, obtained with EMC3EIRENE, which simulates a detachment discharge with RMP application (\#85946). In the figure, the Langmuir probe measurements at inboard and upper divertor plates are also shown. In the simulation, the input power into the SOL $\left(\mathrm{P}_{\mathrm{SOL}}\right)$ and the density at the LCFS are matched with the experiment. The perpendicular transport coefficients for particle and energy transport are set to $D=\chi / 3=1.0 \mathrm{~m}^{2} / \mathrm{s}$. The impurity is carbon that originates from the divertor plates with sputtering coefficient of $1 \%$. The detachment transition is identified with a sudden decrease in the divertor flux measured by the probe at $\sim 4.5 \times 10^{19} \mathrm{~m}^{-3}$. In the simulation the rollover of the divertor flux occurs at similar density, $5.5 \times 10^{19} \mathrm{~m}^{-3}$, where the volumetric energy loss via impurity

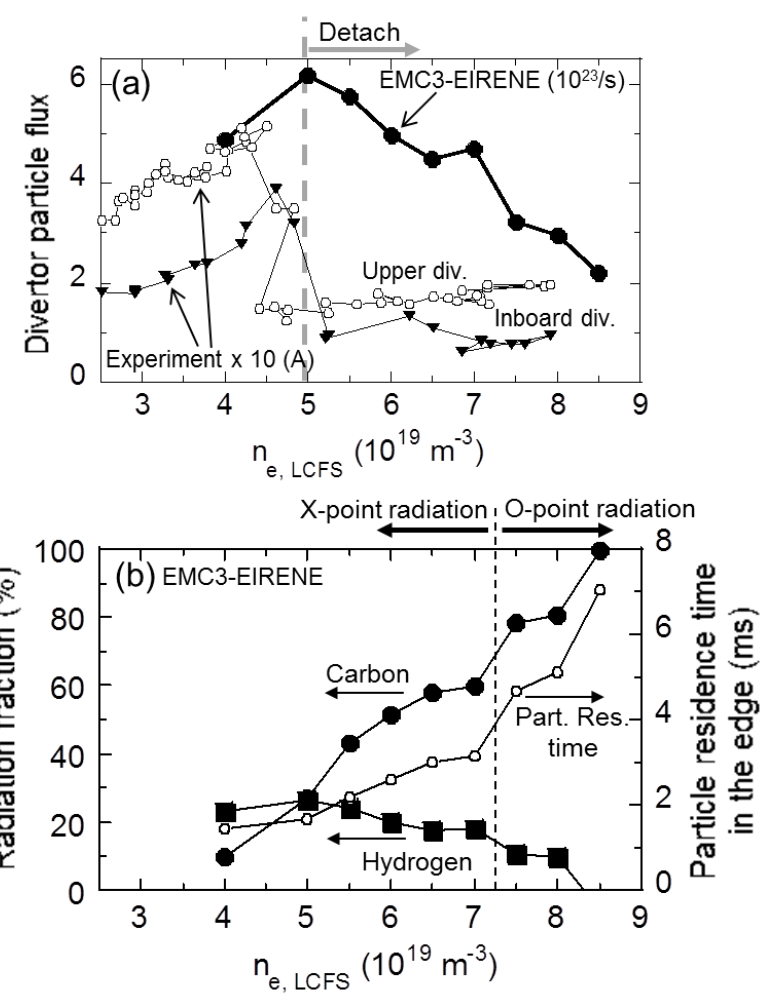

Figure 8. (a) Evolution of divertor particle flux obtained with EMC3-EIRENE and experiments, as a function of $\mathrm{n}_{\mathrm{LCFS}}$, for the detachment shot with RMP (\#85946). $\circ$ and $\boldsymbol{\nabla}$ represent upper and inboard divertor probe measurements, respectively. (b) Density dependence of the radiated power and the averaged particle residence time in the computation domain, obtained with EMC3-EIRENE.

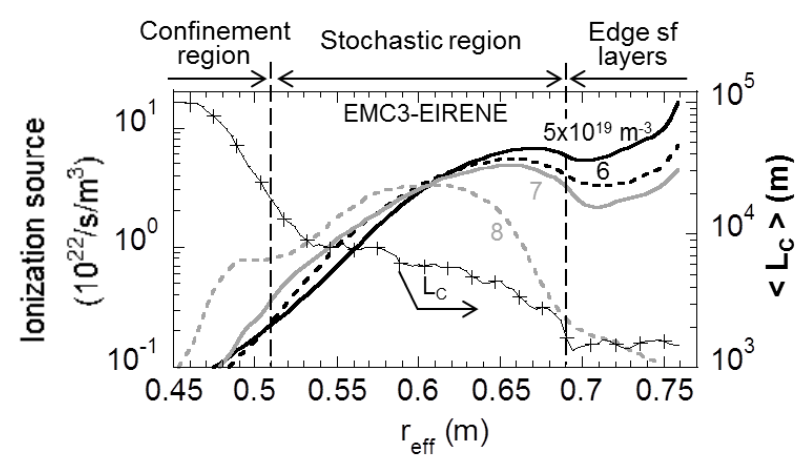

Figure 9. Radial profiles of ionization source for densities at $\mathrm{n}_{\mathrm{LCFS}}=5,6,7$, and $8 \times 10^{19} \mathrm{~m}^{-3}$, obtained with EMC3-EIRENE for the case of Fig. 8 . The volume averaged connection length profile, $\left\langle\mathrm{L}_{\mathrm{C}}\right\rangle$, is shown on the right axis. The edge region is divided into three domains characterized with the change in $\left\langle\mathrm{L}_{\mathrm{C}}\right\rangle$. 
(carbon) plus hydrogen exceeds $50 \%$ of $\mathrm{P}_{\mathrm{SOL}}$, as shown in Fig. 8 (b). Before the rollover, there is no high-recycling behavior observed, as investigated in detail in Refs. [28, 65, 67]. As the density increases further, the divertor flux decreases gradually. However, the reduction in the calculated total recycling flux is much slower than the locallymeasured ion saturations currents. Although the volume recombination process is not implemented in the simulation, the downstream temperature is still more than $1 \mathrm{eV}$ until $\mathrm{n}_{\mathrm{LCFS}}=7.0 \times 10^{19} \mathrm{~m}^{-3}$, in which case the process is considered negligible. The reason for the discrepancy in the divertor particle flux is not yet understood.

On the other hand, the evolution of the divertor particle flux is interpreted as being closely related to the edge magnetic field structure. Figure 9 shows the radial profiles of the ionization source obtained with EMC3-EIRENE for different densities, together with the connection length $\left(\mathrm{L}_{\mathrm{C}}\right)$. The $\mathrm{L}_{\mathrm{C}}$ is volume-averaged in the poloidal and toroidal directions. The clear stepwise increases in $L_{C}$ at $r_{\text {eff }}$ $=0.69$ and $0.51 \mathrm{~m}$ indicate a transition of the magnetic field structure. The region with $r_{\text {eff }}>0.69$

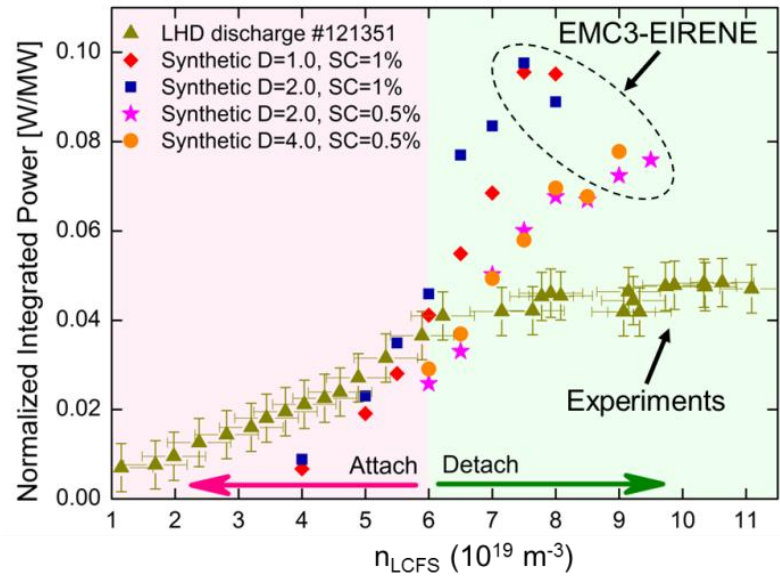

Figure 10. Density dependence of the radiated power intensity obtained by experiments (\#121351) and EMC3-EIRENE. The perpendicular impurity transport coefficient, $\mathrm{D}_{\text {imp }}$, and the carbon sputtering coefficient, $\mathrm{SC}$, are scanned. The power is integrated over the field of view and normalized with $\mathrm{P}_{\mathrm{SOL}}$. Adopted from ref.[68].

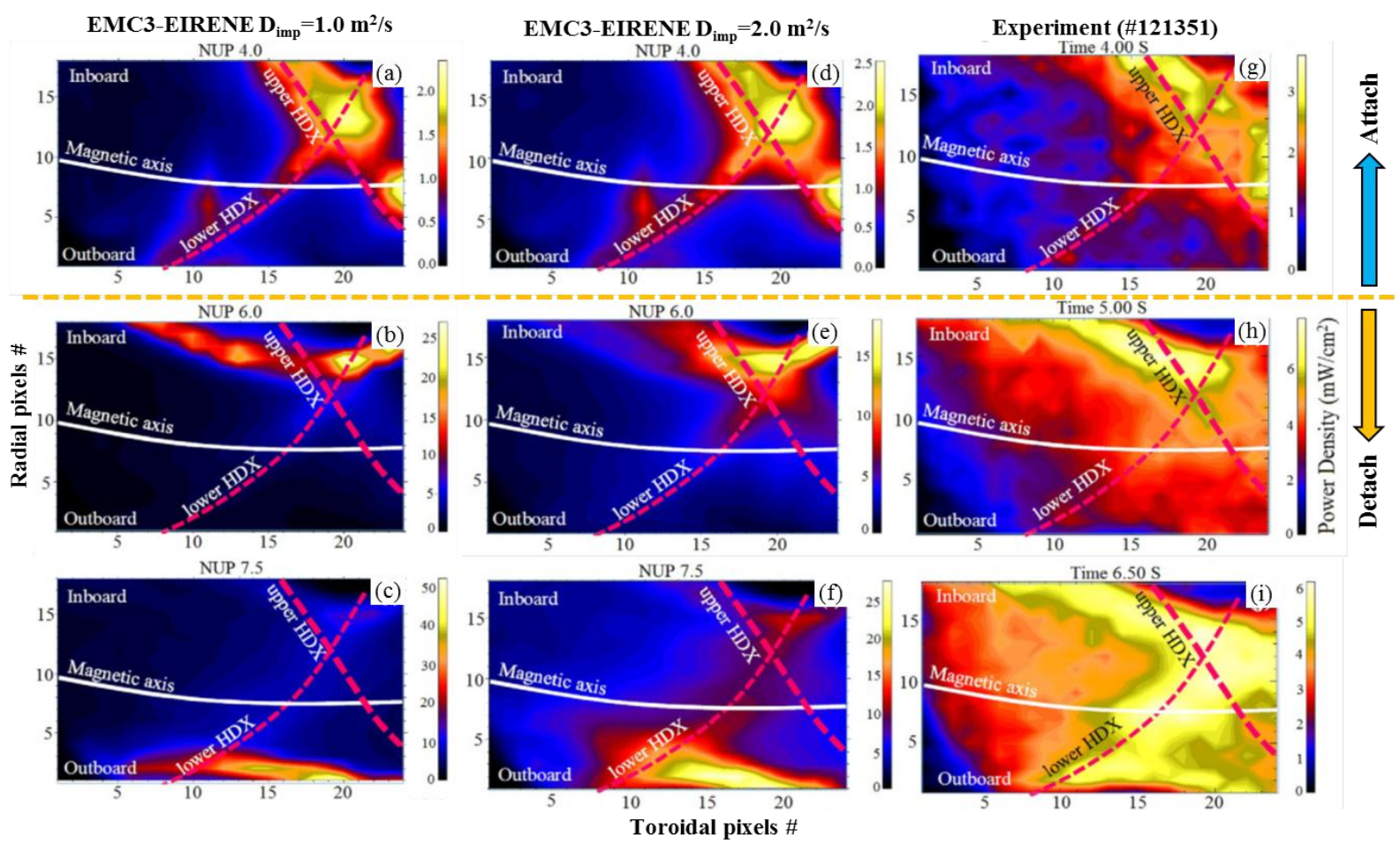

Figure 11. Radiated power distribution by experiments and EMC3-EIRENE in the field of view of IRVB measurements. The first column $(\mathrm{a}, \mathrm{b}, \mathrm{c})$ and the second column $(\mathrm{d}, \mathrm{e}, \mathrm{f})$ represent the synthetic images of EMC3-EIRENE for $\mathrm{D}_{\mathrm{imp}}=1.0$ and $2.0 \mathrm{~m}^{2} / \mathrm{s}$, respectively. The third column $(\mathrm{g}, \mathrm{h}, \mathrm{i})$ is obtained with the IRVB (\#121351). Density increases from the top to bottom rows, and the detachment transition occurs after the first row. Adopted from ref.[68]. 
$\mathrm{m}$ is the edge surface layer [3], where short and long flux tubes co-exist, which is also known as the laminar region [66]. In this region SOL-like parallel transport dominates due to the flow acceleration caused by the sink action at the divertor plates. It is also noted that the short flux tube volume is fed with plasma particles and energy by the long flux tubes via perpendicular transport, and thus allows fast escape of the plasma towards the divertor plates. The region with $0.51 \mathrm{~m}<\mathrm{r}_{\text {eff }}<0.69 \mathrm{~m}$ is the stochastic region, which is filled with the longer flux tubes, and the perpendicular transport competes with the parallel transport [67]. The region with $r_{\text {eff }}<0.5 \mathrm{~m}$ is considered as a confinement region with rather long $\mathrm{L}_{\mathrm{C}}>10^{4} \mathrm{~m}$. At the low density the ionization profile is peaked at the edge surface layer. As the density increases, the ionization profiles gradually shift radially inward. This radial shift of the ionization source leads to an effective increase in the particle residence time in the edge region because of the change of transport features between the edge surface layer and the stochastic region, as mentioned above and also pointed out in [65]. This is seen in Fig.8 (b), where the averaged particle residence time is plotted. At $\mathrm{n}_{\mathrm{e}}=8 \times 10^{19} \mathrm{~m}^{-3}$ there is a sudden penetration of the ionization front into the stochastic region, as seen in Fig.9, where the peak location completely shifts to the stochastic region. This results in a sharp increase in the residence time as shown in Fig.8 (b). The penetration is also accompanied by a sudden decrease in the divertor flux as well as an abrupt increase in the radiated power as shown in Fig.8 (a) and (b). The latter is attributed to the change of edge radiation distribution with respect to the $\mathrm{m} / \mathrm{n}=1 / 1$ magnetic island, that is, the movement of the radiation peak from the $\mathrm{X}$-point to the $\mathrm{O}$-point, as shown below in the comparison with experiments and also discussed in ref.[68]. The simulation results indicate selective cooling at the $\mathrm{X}$ - or $\mathrm{O}$ points of the magnetic island [64, 68]. These results show the important role of the magnetic field structure on the evolution of divertor particle flux and radiated power distribution at the detachment transition.

The absolute intensity of the radiated power has been investigated by comparing the simulation results with the IRVB (infrared imaging video bolometer) measurement [69]. The IRVB views the plasma from the top of the torus and detects the radiation in the wavelength range of $0.15 \sim 1240 \mathrm{~nm}$. The measured power is integrated over the field of view and plotted as a function of density in figure 10, together with the results from the corresponding synthetic diagnostic of EMC3-EIRENE [68]. In the simulation, the database in the STRAHL module is used to calculate the total carbon radiation. The contributions of radiation from the core region due to the high- $Z$ impurity iron and
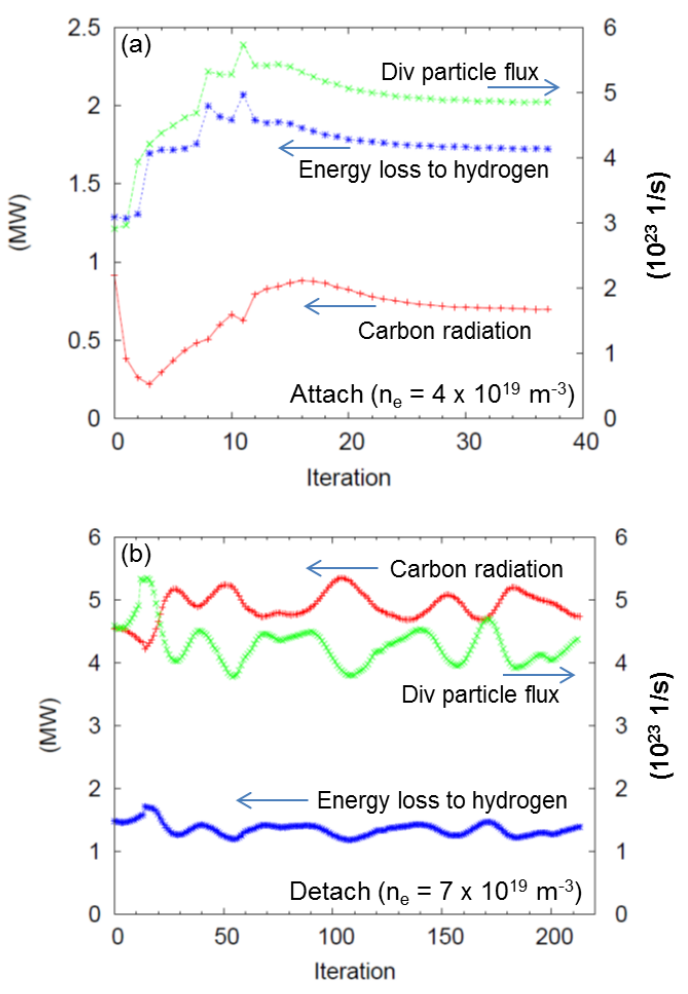

Figure 12. Evolutions of global physical quantities in the EMC3-EIRENE computations, as a function of iterations. (a) The attached case and (b) the detached case. Divertor particle flux (right axis), energy loss to hydrogen, and radiated power via carbon are shown. bremsstrahlung are found to be small as analyzed in Ref.[68]. During the attached phase the 
simulation tends to underestimate the radiated power, while in the detached phase the simulation leads to an overestimate by a factor up to 2.5. The scan of the perpendicular transport coefficient of impurity $\left(D_{\text {imp }}\right)$ is found to be almost irrelevant in terms of the radiation intensity. Another work on the impurity transport indicates a carbon sputtering coefficient of $0.5 \%$ in the LHD [29]. The use of this value in the computation reduces the power by a factor of 2 , which is still higher than the experiments at the high-density range of $\sim 9 \times 10^{19} \mathrm{~m}^{-3}$. Further assessment is necessary to clarify the reason for the discrepancy.

The radiation distributions are compared between the IRVB and the simulation in Fig.11. The simulation results with $D_{i m p}=1.0$ and $2.0 \mathrm{~m}^{2} / \mathrm{s}$ are presented in the first and second columns, and the IRVB measurements are in the third column. The density increases from the upper to the lower rows. The first (top) row is for the attached phase $\left(\mathrm{n}_{\mathrm{LCFS}}=4.0 \times 10^{19} \mathrm{~m}^{-3}\right.$ ), while the second (middle, $\mathrm{n}_{\mathrm{LCFS}}=6.0 \times 10^{19} \mathrm{~m}^{-3}$ ) and the third (bottom, $7.5 \times 10^{19} \mathrm{~m}^{-3}$ ) rows are for the detached phase. The upper and lower regions in each figure correspond to the inboard and outboard sides of the torus. The trajectories of the X-points of the divertor legs (the helical divertor X-point, not the X-point of the island) are indicated in the figure. Details of the correspondence with the magnetic field structure are described in ref.[68]. In the attached phase, the radiation peaks in the upper right corner, where the $\mathrm{X}$-point of the $\mathrm{m} / \mathrm{n}=1 / 1$ island is located, and the radiation extends along the divertor leg $\mathrm{X}$-point, as shown in Fig.11 (g). This pattern is qualitatively well reproduced in the simulation, while the increased $D_{\text {imp }}$ agrees better with the experiments with a broader distribution of the radiation. The results suggest selective cooling at the X-point of the island. In the detached phase, the radiation becomes intense in the upper right corner (i.e. the X-point of the $m / n=1 / 1$ island) and extends along the inboard side flux surface. Note that the scale of the color bars change greatly from the attached to the detached phases. The experiment shows much broader distribution of the radiation than the simulation. The increase of $D_{\text {imp }}$ up to $4 \mathrm{~m}^{2} / \mathrm{s}$ in the simulation did not lead to further broadening of the radiation. In the bottom row, the simulations show a shift of the radiation peak to the bottom part of the field of view, which corresponds to the O-point of the $\mathrm{m} / \mathrm{n}=1 / 1$ island. This transition is accompanied by an inward shift of the ionization front as discussed above, as shown in Fig.9. The IRVB measurement shows an extension of the radiation toward the bottom part, but still a substantial amount of radiation remains on the
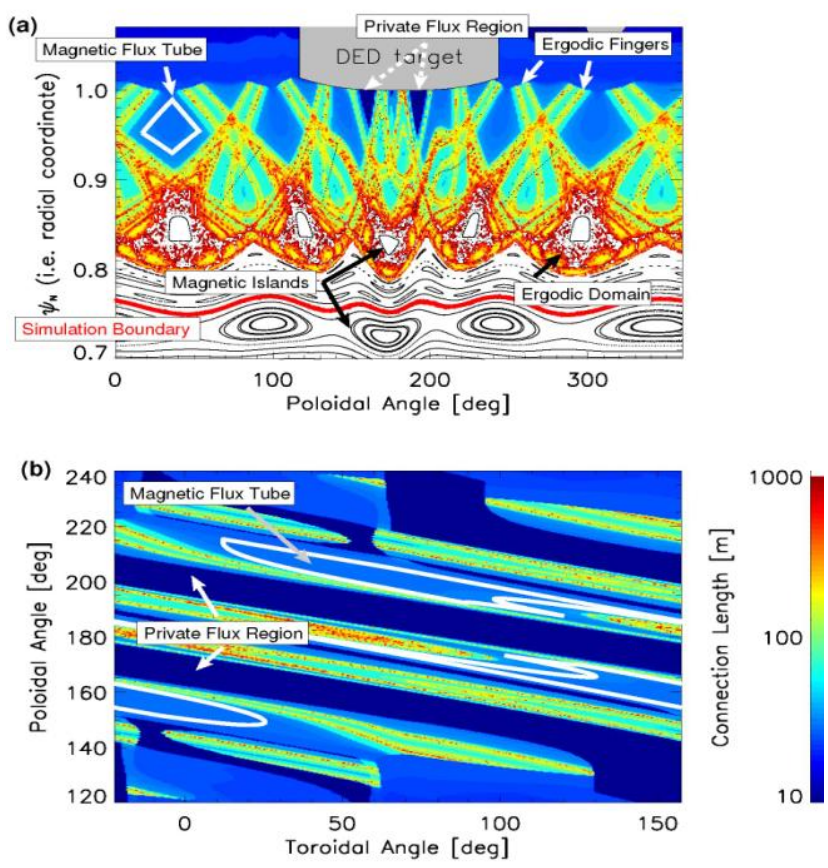

Figure 13. (a) Cross section of the magnetic field structure in the $\mathrm{m} / \mathrm{n}=6 / 2$ helical divertor configuration at TEXTOR-DED. The target to target connection length of field lines allows one to visualize the field structure in the open-field-line region, which is complemented by a Poincare' plot (black) that highlights the ergodic and closed flux surface regions. (b) The corresponding footprint on the DED target. 
inboard side.

These comparisons of the divertor particle flux, radiation intensity, and the distribution suggest a need for further improvement in the transport model of the background plasma as well as that of the impurity in order to reproduce and interpret the detachment processes in stellarators.

When the plasma enters the detached phase, it is found that the computation becomes unstable. This is possibly due to the strong non-linearity of atomic processes in the low temperature range below $\sim 10 \mathrm{eV}$, such as impurity radiation and the ionization of hydrogen, etc. Figure 12 shows the evolution of the global physical quantities in the computations as a function of the iteration. In the attached case, the computation smoothly converges to equilibrium after 30 iterations, as shown in Fig.12 (a). In the detached case, however, the parameters begin to oscillate so that the rise in the carbon radiation leads to the suppression of the divertor particle flux and of the energy loss to the hydrogen and vice versa, as shown in Fig.12 (b). The oscillation persists even after 200 iterations. In the experiments, indeed, one also observes various kinds of fluctuations in the plasma parameters in the detached phase. The oscillation in the computations may come from a certain physical process related to the energy balance between the plasma and impurity. The cause of the oscillation, i.e., whether it originates in a numerical artifact or is related to a physical process, has been discussed and a stabilization of the oscillation has been attempted with an adaptive relaxation scheme [70].

\subsection{Tokamaks}

Tokamak applications of the EMC3-Eirene code have gained significant interest over the last decade, because the code allows one to address the symmetry breaking nature of resonant magnetic perturbations (RMPs) in detail. Today, RMPs are considered to be an essential tool for ELM control in ITER [8], yet the implications for detached divertor operation remain unresolved. But even before recognizing their beneficial impact on ELM control, RMPs have been investigated for their ability to control particle and heat exhaust by the formation of 'ergodic divertors', e.g. in Tore Supra [71] and TEXTOR [72]. Following earlier applications to RMP configurations at TEXTOR [31], present applications of the EMC3-Eirene code include RMP configurations at DIIID [33], ASDEX-U [38], NSTX [35], and extrapolation to ITER [43].

The application of RMPs results in the formation of magnetic island chains (typically in the edge region for which the resonances have been chosen). Neighboring island chains begin to overlap with increasing perturbation strength and form a layer characterized by chaotic (often referred to as stochastic or ergodic) field line behavior. The upstream location is not well defined in these configurations because of the complex 3D structure of the resulting multiple parallel flow channels. This makes the analysis of recycling characteristics challenging, and averaged quantities on the last closed flux surface (LCFS) must serve as proxies for upstream parameters.

RMPs act as a supplement in poloidal divertor configurations in which the particle and heat load areas are still mainly determined by the separatrix strike points produced by the poloidal fields. However, they completely alter the plasma-surface interaction domain in limiter machines such as TEXTOR in such a way as to form edge configurations similar to the divertor configurations explored in stellarators. Thus, these configurations should be treated separately. We will give a brief introduction to the dynamic ergodic divertor (DED in TEXTOR) below in section 3.2.1 and present an overview of the resulting recycling characteristics. Then we move on to the poloidal divertor configuration with RMPs in section 3.2.2. 


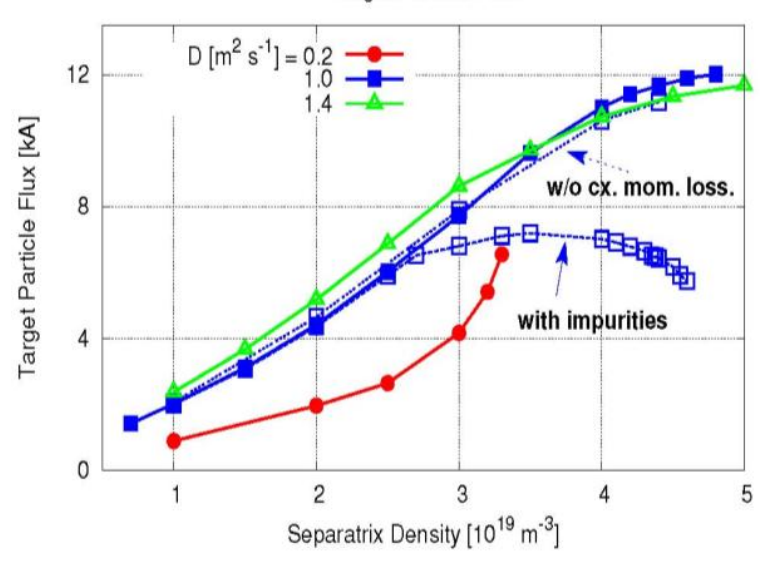

Figure 14. Total recycling flux for three levels of anomalous cross-field transport. Additional simulations have been performed in which charge exchange momentum losses have been turned off (dotted line) and in which impurities have been included (dashed line).

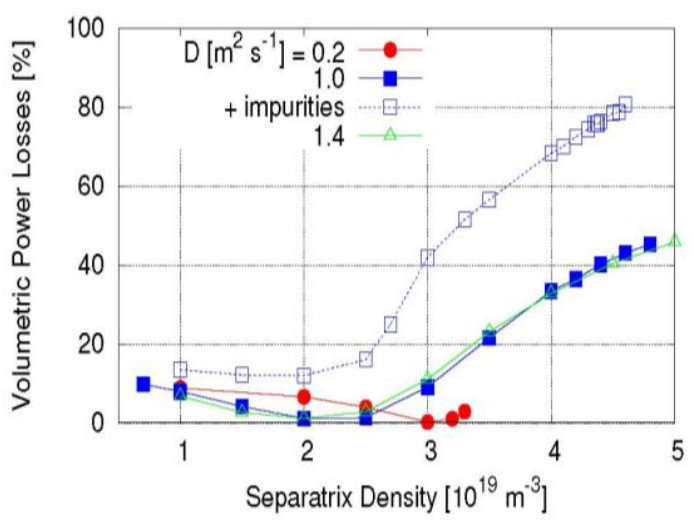

Figure 15. Volumetric power losses (relative to $\mathrm{P}_{\mathrm{SOL}}$ ) from radiation and ionization of neutral hydrogen and impurities.

3.2.1. TEXTOR-DED. The dynamic ergodic divertor (DED) at TEXTOR introduces a nearly helical field divertor configuration [73]. The edge layer is characterized by a mix of shortconnection-length flux tubes and long-connection-length field lines that form finger-like structures. This structure is visualized in figure 13 (a). The footprint is determined by the helical winding of the DED coils which can be operated in base modes with poloidal and toroidal mode numbers $\mathrm{m} / \mathrm{n}=3 / 1$, $6 / 2$ and 12/4. Helical strike lines are generated on the DED target surface separated by private flux regions as can be seen in figure 13 (b). Particle and heat loads follow these helical patterns [32].

Dedicated experimental studies for the $\mathrm{m} / \mathrm{n}=6 / 2$ configuration have shown that the recycling flux increases linearily with increasing density, without transition into a high recycling regime. An 'onset' of detachment can be observed before a radiation instability leads to the formation of a helical divertor MARFE [74] without a stable detachment phase in between. This 'onset' is characterized by a small reduction of the particle flux of a few percent rather than a significant drop as observed during 'traditional' detachment in poloidal divertor tokamaks. Also, the target temperature decreases only to $10-20$ $\mathrm{eV}$, which is still much higher than in 'traditional' detachment and indicates that volume recombination does not play a role. The $3 / 1$ configuration, on the other hand, features a larger divertor volume (and thus increases the ability to screen neutral particles), and high recycling behavior has indeed been observed in those experiments before the plasma falls into a

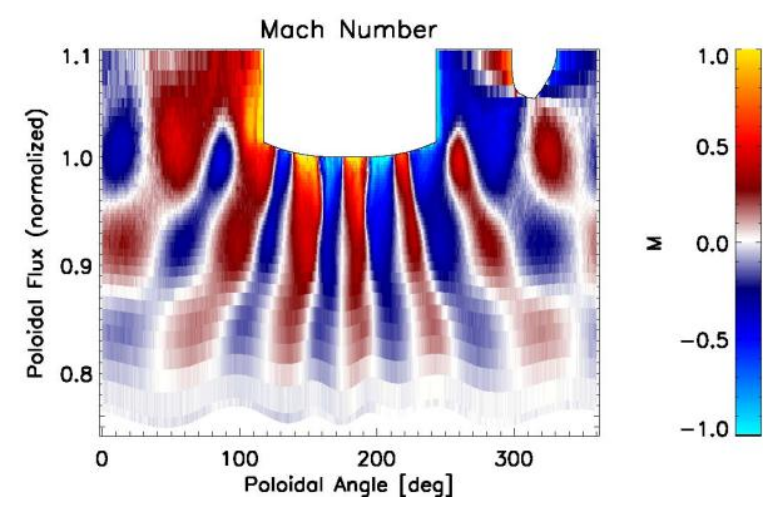

Figure 16. Plasma flow in the TEXTOR helical divertor configuration depicted by the parallel Mach number. 
similar MARFE-like thermal instability [75].

Dedicated numerical studies with EMC3-Eirene have been performed for the $\mathrm{m} / \mathrm{n}=6 / 2$ configuration in figure 13 [76]. The SOL input power in these simulations is set to $\mathrm{P}_{\mathrm{SOL}}=600 \mathrm{~kW}$ based on experimental conditions, and anomalous cross-field heat conduction is fixed at $\chi=3 \times \mathrm{D}$. A significantly different recycling behavior has been found for low and high cross-field diffusion as can be seen in figure 14: at low $\mathrm{D}$, a clear high recycling regime is found in the simulations, while a linear increase with a saturation of the recycling flux at highest densities as seen in the experiment is found only for large enough $D \geq 1 \mathrm{~m}^{2} \mathrm{~s}^{-1}$.

Momentum losses due to charge exchange between hydrogen atoms and ions have been ruled out as a relevant mechansim for the flux saturation at high densities and the suppression of high-recycling (dotted line in figure 14. A rollover of the particle flux (albeit a

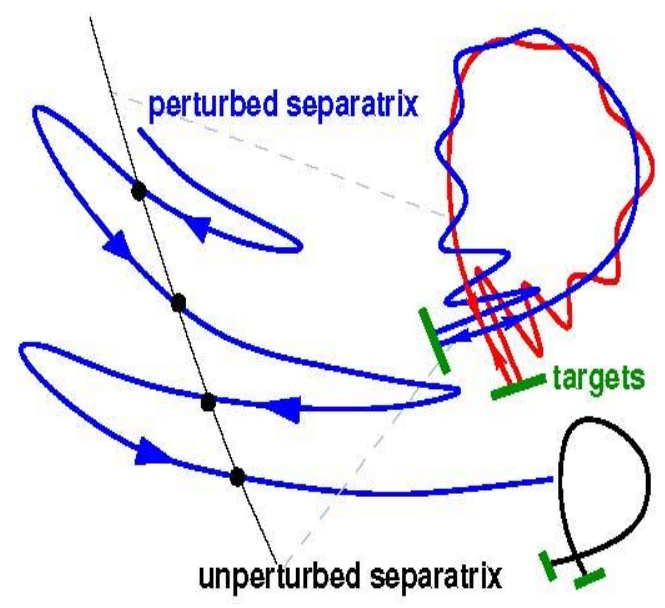

Figure 17. Sketch of the perturbed separatrix manifolds (red and blue). These oscillate around the unperturbed separatrix (black), and can intersect divertor targetes.

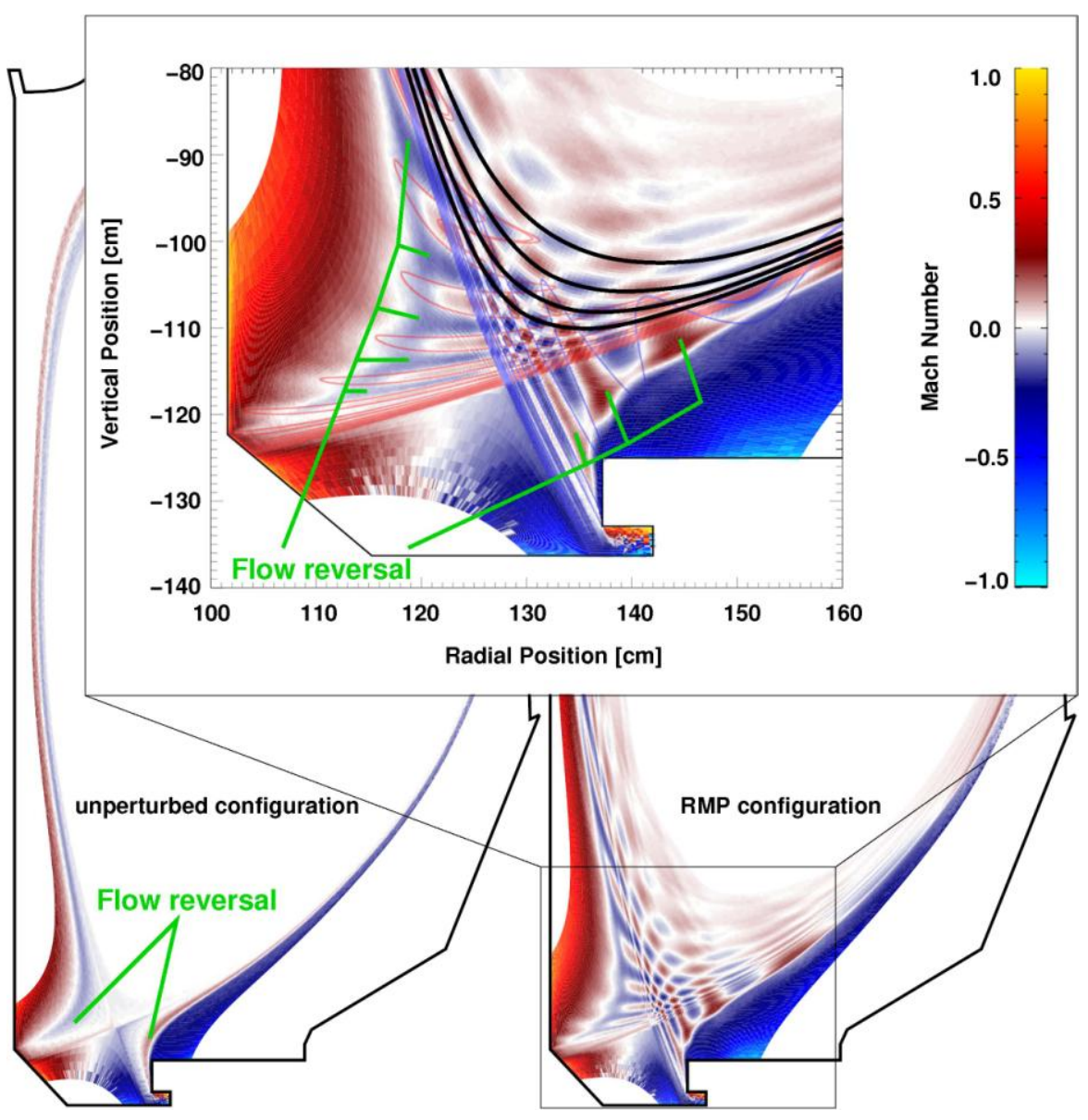

Figure 18. Flow pattern in an axisymmetric (unperturbed) configuration and one with RMPs in DIII-D. 
modest one) has been found after inclusion of carbon impurities, which are released when the plasma comes into contact with the graphite plates on the DED target. A fixed sputtering coefficient of $\mathrm{c}_{\text {sput }}=$ $2 \%$ was chosen for the present simulation. The recycling flux begins to saturate at a separatrix density of $\mathrm{n}_{\text {sepx }} \sim 3.0 \cdot 10^{19} \mathrm{~m}^{-3}$ (the separatrix is approximated here by the inner simulation boundary, which is marked red in figure 13) when impurities are present in the simulations, and only begins to saturate at $\mathrm{n}_{\text {sepx }}=4 \cdot 10^{19} \mathrm{~m}^{-3}$ otherwise. This "onset of detachment" (saturation or slight reduction in the recycling flux) is related to strong volumetric power losses, as can be seen in figure 15, while temperatures remain too high for volume recombination to be relevant [76]. Impurity radiation can provide a significant contribution to the power losses, which prevents the increase of the recycling flux at lower densities.

The presence of counter-flow channels (see figure 16) suggests that a similar mechanism for the suppression of high-recycling and early transition to detachment holds as for W7-AS. However, an estimation of the momentum transfer between two adjacent counter-flow channels shows that friction between counter-flows does not contribute much to the parallel momentum balance in the TEXTOR helical divertor configuration because of the large poloidal size of the relevant edge islands [76]. On the other hand, the presence of significant upstream particle sources, causing an increase of parallel heat convection and a drop of the upstream temperature, has been identified as the underlying mechanism.

\subsubsection{Poloidal divertor configurations with RMPs.}

Detachment in poloidal divertor tokamaks is charac-

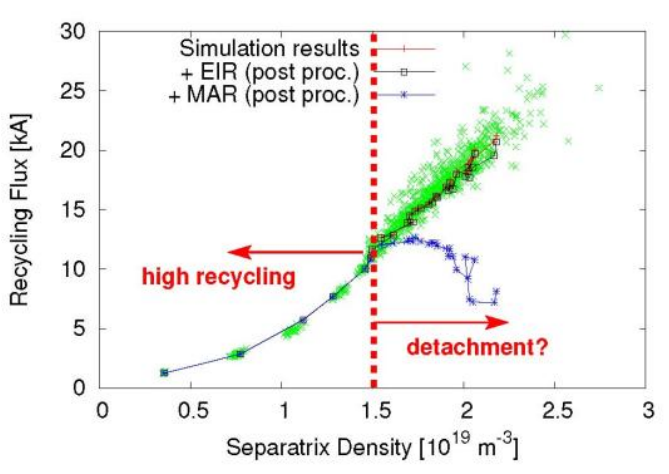

Figure 19. Total recycling flux in an axisymmetric DIII-D configuration (plus sign). For each $\mathrm{n}_{\text {sepx }}, 32$ individual iterations are shown (cross). Corrections from electron-ion recombination (EIR, square) and molecular assisted recombination (MAR, sextile) have been calculated in a postprocessing step.

terized by large gradients in the total plasma pressure parallel to the magnetic field with a reduction of heat and particle loads onto divertor targets [77]. Presently, it is a key element for the ITER reference divertor scenario. Divertor temperatures of $\mathrm{T}_{\mathrm{d}} \leq 5 \mathrm{eV}$ are typically considered to be a necessary (but not sufficient) requirement for detachment [78], although not being a necessary condition for reducing the particle flux in sellarators and ergodic divertor tokamaks. In view of the large geometric differences, different mechanisms can be expected to be relevant for detachment in poloidal divertor tokamaks and stellarators. One expects from a simple two-point analysis for tokamaks that both volumetric power and momentum losses are required to explain detachment [79]. In addition to plasma-neutral friction and radiation, volume recombination can also contribute to both processes. Since the momentum loss resulting

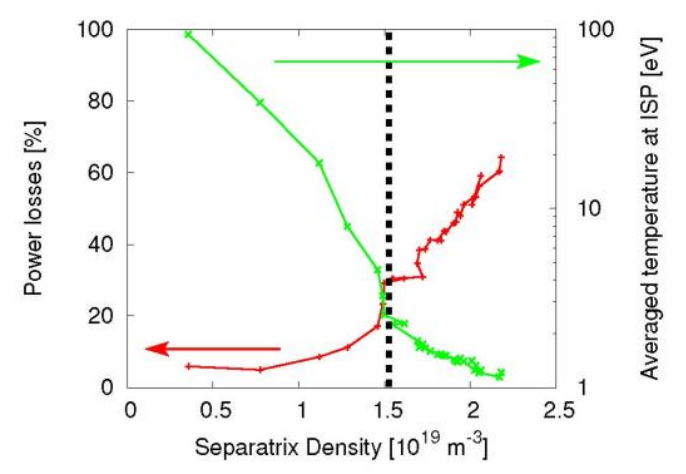

Figure 20. Relative power losses due to plasma-neutral interaction and target temperature at the inner strike point region. 
from plasma-neutral friction depends on neutral transport associated with the realistic divertor geometry, there is no simple theory able to show that, in practical $2 \mathrm{D}$ and $3 \mathrm{D}$ cases, this plasmaneutral interaction can really cause sufficient momentum losses to drive a rollover of the particle flux onto the targets. Although logically not necessary, it appears that volume recombination might be required as shown in a recent review on detachment [80]: the particle flux does not roll over but virtually saturates in SOLPS simulations for a DIII-D-like configuration when recombination is switched off. Similar results are also found in simulations for an ASDEX Upgrade configuration [81] in which volume recombination is required to produce the necessary number of neutral particles for creating sufficient momentum loss.

Of particular interest for the next step fusion device ITER is the compatibility of RMPs (for ELM control) with detached diveror operation. As a result of RMP application, the magnetic separatrix splits into two distinct manifolds, which are characterized by a helical lobe structure (see figure 17). The separatrix manifolds provide an envelope for field lines that connect the plasma interior to the divertor targets, which provides a transport channel for particles and energy in addition to the regular SOL. This can modify the upstream-to-downstream pressure balance, and can thus affect detached divertor operation. It certainly affects the plasma flow in the edge region, which has been investigated in detail by simulations for DIII-D [82]. Two effects can be observed (see figure 18): (1) flow channels with alternating direction are generated on resonant surfaces inside the separatrix and (2) flow reversal in the regular SOL alternates poloidally with plasma flow towards divertor targets inside the perturbed separatrix manifolds. The alternating flows on the resonances are always directed outwards. It can be expected that friction between these flow channels will be of increasing importance towards the separatrix, because their size (and consequently their separation) becomes smaller and smaller both radially and poloidally. This is due to the divergence of the safety factor, which results in a decreasing radial distance between resonances and an increasing poloidal mode number. Flow reversal effects are discussed for the regular SOL and attributed to the presence of strongly-localized sources downstream [79]. This flow reversal can also be generated in the presence of RMP fields, but with a more complex structure: it can be seen in figure 18 that the flow reversal is maintained in the regular SOL (outside the separatrix manifolds), but lost inside the separatrix manifolds due to the higher upstream pressure. To understand how these flows interact with each other and influence the pressure balance, and how this may affect the transition to detachment is an ongoing subject.

It has been found that the onset of detachment is governed by the ratio of the upstream plasma pressure to the specific energy flux into the recycling region [80], however, the upstream location is not uniquely defined in configurations with RMPs, which makes such an analysis challenging for RMP configurations. Averaged quantities on the last closed flux surface (LCFS) may serve as proxies, but it should be noted that not all field lines that are guided onto the divertor targets by the separatrix manifolds connect all the way inside to the LCFS. Also, it must be noted that the 'plasma response' to RMPs will likely result in a different magnetic field structure than obtained from simply overlaying the equilibrium magnetic field and the vacuum perturbation field, and will result in a different radial location of the LCFS.

Unfortunately, the low-temperature high-density divertor regime has so far proven to be very challenging to access with the EMC3-Eirene code: numerical oscillations in the iterated plasma state can be excited (see next section). These oscillations can be related to the iterative approximation of a 
(steady-state) solution of a non-linear system [70], and are not necessarily an indication for a physically unstable plasma. Whether a refinement of physical processes in the model improves this situation remains an open question. Nevertheless, temporal oscillations in the divertor plasma have indeed been observed experimentally [83] and in time-dependent computer simulations [84, 80], and these could provide a problem for the intrinsic steady-state assumption in the EMC3-Eirene framework.

3.2.3. Technical challenges and further development. Traditional tokamak configurations are axisymmetric, to which two dimensional computational models such as SOLPS (B2-EIRENE) [85] and EDGE2D-EIRENE [12] apply. Benchmarks of EMC3-Eirene against these 2D codes have been performed for attached conditions with reasonable agreement $[34,86]$, and it would be of great value to continue this process towards detached divertor conditions. As a start, we explore numerical access to detachment in poloidal tokamaks with EMC3-Eirene based on an axisymmetric configuration.

Simulation results for the total recycling flux $\Gamma_{\text {rec }}$ in an axisymmetric DIII-D configuration are shown in figure 19. It can be seen that $\Gamma_{\text {rec }}$ continues to increase linearily with the separatrix density after an initial non-linear high recycling phase. These simulations have been performed without volume recombination, which may explain the absense of a roll-over. As shown in figure 19, a classical roll-over of $\Gamma_{\text {rec }}$ is not found in the simulations, except for a slope change of the $\Gamma_{\text {rec-curve }}$ at $\mathrm{n}_{\text {sepx }}=1.5 \cdot 10^{19} \mathrm{~m}^{-3}$ accompanied by a sudden increase of power losses from plasma-neutral interaction and a jump of $\mathrm{T}_{\mathrm{d}}$ down to $\sim 2 \mathrm{eV}$ (see figure 20)

A troublesome issue encountered here is that the simulations start to become unstable, which is indicated in figures 19 by plotting the last 32 iterations for each $\mathrm{n}_{\text {sepx }}$ value. The target temperature (averaged over the inner strike point region) suddenly drops to a few eV at $\mathrm{n}_{\mathrm{sepx}}=1.5 \cdot 10^{19} \mathrm{~m}^{-3}$. From this point on both divertor temperature and density begin to oscillate. It has been demonstrated that these kinds of oscillations can be reconstructed within an iterated two-point model [70], which indicates that they might be related to the numerical framework of EMC3-Eirene. An adaptive relaxation scheme has provided some success for stabilization, although it is not yet fundamental enough to guarantee convergence for more than a few example cases. Narrowing down the trigger for these oscillations is part of an ongoing analysis.

Since volume recombination is considered to play a major role in poloidal divertor detachment, we have analyzed the relevance in our simulation results. Two different recombination processes are examined separately: 1) the electron-ion recombination (EIR) including both radiative and three-body recombination and 2) a molecular assisted recombination (MAR) process via the reactions of

$$
\mathrm{H}_{2}+\mathrm{H}^{+} \rightarrow \mathrm{H}_{2}^{+}+\mathrm{H} \text { and } \mathrm{H}_{2}^{+}+\mathrm{e} \rightarrow 2 \mathrm{H} \text {. }
$$

The effective MAR rate coefficient is taken from AMJUEL (reaction 3.2.3r). The respective total recombination rates from the EIR and MAR process are estimated by post-processing the EMC3Eirene data and then subtracted from the total recycling flux $\Gamma_{\text {rec. }}$. The expected net/corrected particle fluxes are shown in figure 19 in comparison with the original ones without recombination. It can be seen in figure 19 that the EIR does not significantly contribute to the particle balance yet in the target temperature range of $1-2 \mathrm{eV}$. However, the MAR would be strong enough to result in a roll-over in $\Gamma_{\text {rec. }}$ Whether this holds in self-consistent simulations remains to be verified. Spectroscopic 
measurements indicate that MAR could be important during detachment in DIIID [88], although it is found to be not as dominant as indicated in figure 19. Nevertheless, it underlines the importance of benchmarking the code behavior in this low-temperature range.

\section{Discussion}

3D divertors in tokamaks and stellarators share a common geometric feature - the existence of resonant helical field components. Typical examples are the island divertor for W7-AS and W7-X, the helical divertor of LHD, the "ergodic" divertors explored at Tore Supra and TEXTOR and the RMP-modified poloidal divertor on tokamaks. Helical fields are a natural product of the 3D-shaped coils in stellarators, while they are produced in tokamaks by applying external coils. In the poloidal divertor, RMP-fields just add a perturbation to the poloidal diverting field, whereas the other 3D divertor concepts completely rely on helical fields. Depending on the spectrum of the helical fields provided in each device, the resulting divertor configuration can differ significantly from device to device, as does the related plasma transport, in particular under detached conditions.

Detached plasmas in W7-AS and LHD have been studied extensively using the EMC3-Eirene code. In the simulations, carbon is assumed to be the main radiator because the divertor targets in both devices are made of carbon. The essential features of the detached plasmas observed experimentally, i.e. the rollover of recycling flux (ion saturation currents) and intensive impurity radiation could be well reproduced by the code. Simulations and experiments have consistently shown that, in both devices, plasmas go into detachment without a prior high-recycling regime and the total plasma thermal pressure (electrons + ions) drops along field lines much more strongly than by the factor of 2 required to accelerate the ions to the sound speed at the sheath entry. This behavior is attributed to cross-field viscous transport of the parallel plasma flows associated with the specific magnetic-field topologies in these devices. This viscous transport drops with decreasing perpendicular viscosity and a high-recycling regime would be expected to appear at sufficiently-low viscosities or for sufficiently large islands as in W7-X. This has been numerically demonstrated in the TEXTOR-DED simulations, where a high-recycling regime could have been found for the $m / n=6 / 2$ configuration if the anomalous transport would have been smaller by a factor of 5 . In these helical configurations plasma-neutral friction does not contribute to the parallel momentum balance considerably, which has been shown by switching off the corresponding terms in dedicated calculations for W7-AS and TEXTOR-DED. The drop of recycling flux in detachment of W7-AS and LHD is consistent with the consideration of an enhancement of the geometry-related momentum loss and of the increased particle residence/transport time in the SOL associated with an inward shift of the ionization front of the recycling neutrals.

Both W7-AS and LHD divertor experiments have shown that stabilizing a detached plasma against thermal instabilities necessitates external perturbation fields in addition to the intrinsic ones. EMC3-Eirene simulations show a close correlation of the radiation distribution with the field structure, or more precisely, with the X-points (also O-points in LHD) of the dominating islands. The predicted radiation locations for stable detached plasmas in W7-AS and LHD qualitatively agree with the experimental tendencies. However, large discrepancies in absolute numbers usually exist between the calculated results and local diagnostics. The absence of drifts in the present code version is considered to be one of the major obstacles that prevent more quantitative studies of detachment in stellarators. Drifts are being implemented in the code. The work has been started with the solving of 
a potential/current equation including the electrostatic potential, the polarization current and an anomalous current caused by a finite cross-field conductivity.

Numerical attempts to access a detachment regime for the poloidal divertor with RMP fail so far. No stable solutions can be found when $T_{e}$ approaches a few electron volts (typically below $5 \mathrm{eV}$ ) at the targets. Computations usually behave unstably in the manner that the simulated plasma oscillates over iterations periodically within limited amplitudes. The reason is not yet understood. The coupled fluid equations and the neutral- and impurity-plasma interaction form a strongly nonlinear system. They are solved in the 3D code in a standard relaxation (iterative) way. Iterationinduced oscillations due to possible positive feedback from the iteration loops should be, in principle, avoidable by carefully selecting the relaxation factor/strategy. This has been attempted but has not fundamentally solved the problem [70]. Thus, parallel effort is also devoted to looking at the physics. Physically, for the conventional poloidal divertor geometry, there is indeed an ionization-related thermal instability in that low- $T_{e}$ range [89]. This unstable region can be removed when there are sufficient momentum losses to reduce the recycling flux. So far, a rollover of recycling flux, as robustly reproduced for stellarators, has not yet appeared in the poloidal divertor simulations with and without RMP, indicating insufficient momentum losses there. Presently, a great effort is being made to reexamine volume recombination processes, in particular a molecular-assisted one.

Similar, but not identical, oscillation phenomena have also been seen in LHD detachment simulations, but where no oscillation periodicity could be identified. It is difficult to justify that this oscillation is not a purely-numerical event. On the other hand, however, multiple stable/unstable solutions could exist for the highly non-linear system under detached conditions in a complex stochastic field with a rich spectrum like in the edge of LHD. If this would be the case, inherent Monte-Carlo noise could potentially drive the system from one solution to another. While these explanations are speculative, one has indeed observed various kinds of fluctuations during the detached phase in LHD experiments.

\section{Summary}

It has been demonstrated that fluid modeling of the edge plasma transport in general 3D magnetic configurations, for which the well-developed finite difference/volume methods are hardly applicable, is feasible via a Monte-Carlo approach in EMC3. In comparison to the most advanced 2D tokamak models, the 3D EMC3 code is still less complete in physics, but much more powerful in geometry. Coupled with Eirene, the EMC3-Eirene code package has been widely applied to most of the divertor configurations/concepts explored on stellarators, tokamaks and linear devices, with most of the numerical achievements still being more qualitative than quantitative.

Detachment physics in W7-AS and LHD has been studied extensively using the EMC3Eirene code. The code has captured essential features of the detached plasmas observed in both devices, i.e. the rollover of recycling flux (ion saturation current), the intensive impurity radiation and the correlation between magnetic field structure and radiation distribution. Significant quantitative discrepancies with local experimental measurements have usually been observed, however. For a more quantitative understanding of detachment physics in stellarators, drift effects are being implemented in the code.

Detachment simulation for the poloidal divertor with RMP-field has been attempted, unfortunately without success so far. Once the target $T_{\mathrm{e}}$ drops down to a few eV (typically below 5 
eV), no stable solution can be found and the plasma instead oscillates within limited amplitudes. A rollover of the recycling flux has never been produced in a stable way. Great efforts are being made to find the underlying numerical and physical reasons. Particular attention is being paid to volume recombination processes.

Benefiting from international collaboration, the $3 \mathrm{D}$ code is being further developed in a parallel way. Major ongoing activities involve the implementation of drift and volume recombination effects and model extension to multiple fluid and kinetic impurities.

\section{Acknowledgement}

This work was supported in parts (H. Frerichs) by the U.S. Department of Energy (DOE) under grants DE-SC0012315 and DE-SC001391 and in parts (M. Kobayashi) by the NIFS budget ULPP026, and by Japan Society for the Promotion of Science Grant Nos. 25420893, 15H04236, and 16H04622. One of the authors (M. Kobayashi) would like to thank the fruitful discussions with Prof. B.J. Peterson, Dr. S.N. Pandya, and Dr. G. Kawamura.

\section{References}

[1] Grigull P et al 2001 Plasma Phys. Control. Fusion 43 A175-A193

[2] Morisaki T et al.,2005 J. Nucl. Mater. 337-339, 154

[3] Ohyabu N et al 1994 Nucl. Fusion 34387

[4] Renner H et al 2000 Nucl. Fusion 401083

[5] Evans T. et al. 2004 Phys. Rev. Lett. 92235003

[6] Liang Y. et al. 2007 Phys. Rev. Lett. 98265004

[7] Suttrop W et al. 2011 Phys. Rev. Lett. 106225004

[8] Loarte A et al 2014 Nucl. Fusion 54033007

[9] Braginskii S I 1965 Transport processes in a plasma Reviews of Plasma Physics vol 1 (New York: Consultants Bureau) pp 205-311

[10] Braams B J 1987 A multifluid code for simulation of the edge plasma in tokamaks NET Report No 68 January 1987 (EUR-FU/XII-80/87/68)

[11] Rognlien T D et al 1992 J. Nucl. Mater. 196-198 347-51

[12] Simonini R et al 1994 Contrib. Plasma Phys. 34 368-73

[13] Schneider R et al 2000 Contrib. Plasma Phys. 40 (3-4) 340

[14] Zagorski R et al 2008 Nucl. Fusion 48024013

[15] Runov D A et al 2001 Phys. Plasmas 8 916-930

[16] Feng Y et al 1999 J. Nucl. Mater 266-269 812

[17] Reiter D et al 2005 Fusion Science and Technology 47172

[18] Feng Y et al 2005 Phys. Plasmas 12052505

[19] Feng Y et al 2004 Contrib. Plasma. Phys. 4457

[20] Frerichs H et al 2010 Comput. Phys. Commun. 181 61-70

[21] Kawamura G et al 2014 Contrib. to Plasma Phys. 54437

[22] Feng Y et al 2014 Contrib. Plasma. Phys. 54426

[23] Feng Y et al 2013 Computer Phys. Communications 1841555

[24] Rack M et al 2016 A hybrid fluid-kinetic approach for 3D plasma edge transport in He-plasma, submitted to Nuclear Fusion

[25] Sharma D et al 2005 Nucl. Fusion 45825

[26] Hölbe H et al 2016 Nucl. Fusion 56026015

[27] Effenberg F et al 2016 Numerical investigation of plasma edge transport and limiter heat fluxes in Wendelstein 7-X startup plasmas with EMC3-Eirene, submitted to Nuclear Fusion

[28] Kobayashi M et al 2010 Fusion Science and Technology 58220

[29] Dai S et al 2016 Nucl. Fusion 56066005

[30] Bader A et al 2013 Nucl. Fusion 52113036

[31] Kobayashi M et al 2004 Nucl. Fusion 44 S64 
[32] Schmitz O et al 2008 Nucl. Fusion 48024009

[33] Frerichs H et al 2010 Nucl. Fusion $\mathbf{5 0} 034004$

[34] Harting D et al 2011 J. Nucl. Mater. 415 S540-4

[35] Lore J D et al 2012 Nucl. Fusion 52054012

[36] Lore J D et al 2015 Phys. Plasmas 22056106

[37] Lunt T et al 2011 Plasma Phys. Control. Fusion 53125010

[38] Lunt T et al 2012 Nucl. Fusion 52054013

[39] Zhang W et al 2016 Nucl. Fusion 56036007

[40] Brida M et al 2016 Determination of the Stochastic Layer Properties Induced by Magnetic Perturbations via Heat

Pulse Experiments at ASDEX Upgrade, submitted to Nuclear Materials and Energy

[41] Kobayashi M 2007 Nucl. Fusion 4761

[42] Zha X et al 2009 J. Nucl. Mater 390-391 398

[43] Schmitz O et al 2016 Nucl. Fusion 56066008

[44] Lunt T et al 2014 Plasma Phys. Control. Fusion 56035009

[45] Huang J. et al 2014 Plasma Phys. Control. Fusion 56075023

[46] Sharma D et al 2013 J. Nucl. Mater. S554-S558 438

[47] Sahoo B P et al 2015 Nucl. Fusion 55063042

[48] Rapp J et al 2015 J. Nucl. Mater. 463510

[49] Kuwabara T et al 2016 Contrib. Plasma Phys. doi: 10.1002/ctpp.201610049

[50] McCormick K et al 2002 Phys. Rev. Letters 89015001

[51] Wagner F et al 2005 Physics of Plasmas 12072509

[52] Hirsch M et al 2008 Plasma Phys. Control. Fusion 50053001

[53] Feng Y et al 2011 Plasma Phys. Control. Fusion 53024009

[54] Feng Y et al 2002 Plasma Phys. Control. Fusion 44611

[55] Feng Y et al 2006 Nucl. Fusion 46807

[56] Grigull P et al 2003 Fusion Engineering and Design 66- 68 49-58

[57] Feng Y et al 2005 Nucl. Fusion 4589

[58] Wenzel U. et al 2002 Plasma Phys. Control. Fusion 44 L57

[59] Thomsen H. et al 2004 Nucl. Fusion 44820

[60] Grigull P. et al 2003 J. Nucl. Mater. 313-316 1287

[61] McCormick K. et al 2005 J. Nucl. Mater. 337-339 520

[62] Wenzel U. et al 2004 Nucl. Fusion 441130

[63] Ramasubramanian N. et al 2004 Nucl. Fusion 44992

[64] Kobayashi M et al 2013 Nucl. Fusion 53093032

[65] Feng Y et al 2008 Nucl. Fusion 48024012

[66] Nguyen F et al 1997 Nucl. Fusion 37743.

[67] Kobayashi M et al 2015 Nucl. Fusion 55104021

[68] Pandya S N et al 2016 Nucl. Fusion 56046002.

[69] Peterson B J 2000 Rev. Sci. Instrum. 713696.

[70] Frerichs H et al 2015 Computer Physics Communications 18882.

[71] Ghendrih Ph and Grosman A 2009 Fusion Science and Technology 561432

[72] Finken K H and. Wolf G H 1997 Fusion Engineering and Design 37337

[73] Finken K H 1997 J. Nucl. Mater. 37583

[74] Clever M et al 2012 Nucl. Fusion 52054005

[75] Lehen M et al 2005 J. Nucl. Mater. 337-339 171

[76] Frerichs H et al 2012 Nucl. Fusion 52023001

[77] Matthews G F 1995 J. Nucl. Mater. 220-222 104

[78] Stangeby P C 1993 Nucl. Fusion 331695

[79] Stangeby P C 2000 The Plasma Boundary of Magnetic Fusion Devices (Plasma Physics Series)(Bristol: Institute of Physics Publishing)

[80] Krasheninnikov S I et al 2016 Phys. Plasmas 23055602

[81] Kotov V and Reiter D 2009 Plasma Phys. Control. Fusion 51115002

[82] Frerichs H et al 2015 Phys. Plasmas 22072508

[83] Loarte A et al 1999 Phys. Rev. Lett., 833657

[84] Smirnov R D et al 2016 Phys. Plasmas 23012503

[85] Schneider R et al 2006 Contrib. Plasma Phys. 46(1-2) 3

[86] Feng Y et al 2011 EMC3-Eirene/SOLPS4.3comparison for ITER. 38th EPS Conference on Plasma Physics, 27 
June- $1^{\text {st July 2011Strasbourg/France P1.071 }}$

[87] Pigarov A Y and Krasheninnikov S I 1996 Phys. Lett. A 222

[88] Hollmann E M et al 2006 Plasma Phys. Control. Fusion 481165

[89] Borras K 1991 Nucl. Fusion 311035 
